\title{
Magnetic form factor, field map, and field distribution for a BCS type-II superconductor near its $B_{\mathrm{c} 2}(T)$ phase boundary
}

\author{
A. Maisuradze ${ }^{1,2}$ and A. Yaouanc ${ }^{2,3}$ \\ ${ }^{1}$ Physik-Institut der Universität Zürich, Winterthuerstrasse 190, CH-8057 Zürich, Switzerland \\ ${ }^{2}$ Laboratory for Muon-Spin Spectroscopy, Paul Scherrer Institute, 5232 Villigen-PSI, Switzerland \\ ${ }^{3}$ Institut Nanosciences et Cryogénie, SPSMS, CEA and University Joseph Fourier, F-38054 Grenoble, France \\ (Received 20 February 2013; revised manuscript received 28 March 2013; published 10 April 2013)
}

\begin{abstract}
We review the magnetic form factor deduced by Delrieu from Gorkov's equation for a Bardeen-CooperSchrieffer (BCS) type-II superconductor near its $B_{\mathrm{c} 2}$ phase boundary, i.e., when its magnetization is small. A numerical study of the form factor, field map, and field distribution follows. The characteristics of the transition from the low-temperature BCS to the high-temperature Ginzburg-Landau vortex lattices is studied. The exotic shape of the component field distribution and the form factor at low temperature and as a function of the external field intensity are discussed. Our numerical work should be helpful for the analyzing of small-angle neutron scattering and muon spin rotation vortex-lattice data recorded for BCS superconductors and may be other superconductors in the clean limit.
\end{abstract}

DOI: 10.1103/PhysRevB.87.134508

PACS number(s): 74.25.Uv, 61.05.fg, 76.75.+i

\section{INTRODUCTION}

The bulk properties of a vortex lattice (VL) of a typeII superconductor are studied experimentally, among other techniques, by magnetization, small-angle neutron scattering (SANS), muon spin rotation ( $\mu \mathrm{SR})$, and nuclear magnetic resonance (NMR) measurements. To extract physical information on the investigated compound modeling of the VL magnetic properties is required. This is usually done using either the London or the Ginzburg-Landau (GL) models. ${ }^{1}$ While the London model neglects the vortex cores altogether-which is acceptable for low fields only-the GL model accounts for them. Although the GL theory is strictly valid only near the superconducting critical temperature at low field it turned out to be a good approximation for a number of classical BCS superconductors. ${ }^{2}$ The GL model is usually found to provide a proper description of the VL throughout the mixed phase for unconventional superconductors also, assuming the London penetration depth and the Ginzburg-Landau coherence length to be effective parameters. ${ }^{3}$ This is further discussed by Landau and Keller. ${ }^{2}$

One of the interesting phenomena predicted already in 1972 by Delrieu is the diffraction of the Cooper pairs on the periodic potential induced by the VL. ${ }^{4}$ He showed that for clean superconductors at low temperatures and fields close to the upper critical field $B_{\mathrm{c} 2}$ exotic behaviors of the VL may be observed due to Cooper pair diffraction. The spatial field distribution around vortex cores obtains a conical shape and the positions of the minimal and saddle-point fields interchange. As a consequence the probability field distribution shows a linear tail around the vortex core field. Nearly at the same time E. H. Brandt came to the same conclusion based on a nonlocal theory of superconductivity. ${ }^{5}$ In the following publications he presented analytical and numerical results for the nonlocal VL behavior at fields close to $B_{\mathrm{c} 2}$ with an arbitrary impurity scattering, ${ }^{6}$ and later for a broad range of fields. ${ }^{7}$ Later on, an exact numerical solution of Eilenberger's quasiclassical equations by Klein allowed one to determine the microscopic structure of the order parameter and magnetic field in the whole range of the applied fields. ${ }^{8}$
Writing a simplified Gorkov ${ }^{9}$ integral equation for the Green's function in terms of a set of linear algebraic equations for the time and space Fourier components, U. Brandt et al. have been able to compute analytically the density of states under high fields. ${ }^{10}$ Later on the magnetization ${ }^{11}$ as well as the field distribution ${ }^{4,12}$ were also obtained by U. Brandt $e t$ al. and Delrieu, respectively, using the previously derived results for the Green's function. The clean and dirty limits were considered by Delrieu in his Ph.D. thesis. ${ }^{12}$

It is worth noting that the Cooper pair diffraction is a property of clean superconductors. Therefore, in most hightemperature superconductors these diffraction effects may be observable.

A VL field distribution which may exhibit a high-field linear tail was reported from NMR measurement on vanadium. ${ }^{13}$ Later on the existence of the tail was confirmed by Herlach et al. for niobium using $\mu \mathrm{SR}$ measurements. ${ }^{14}$ They were performed at low temperature and for an external field $B_{\text {ext }}$ relatively close to $B_{\mathrm{c} 2}$. The linear tail is qualitatively different from GL model expectation for which a field cutoff should be present. ${ }^{15}$

The NMR and the $\mu$ SR results seem to support Delrieu's predictions, in particular the linear tail. SANS data may also be consistent with them. ${ }^{4}$ However, after more than 40 years there is still no definitive experimental observation of the predicted exotic VL at low temperature. Probably the difficulty of reading Delrieu's works has prevented experimentalists from performing the required combined SANS and $\mu$ SR measurements. Here we review this work and present a numerical analysis of the form factor, field map, and field distribution.

The organization of this paper is as follows. Section II recalls the geometry of a VL and introduces useful reduced quantities. In Sec. III the physical principles behind the computations of the magnetization and form factor are given. The following sections, i.e., Secs. IV and V, deal with the magnetization and form factor, respectively. In Sec. VI the component field distribution is discussed. The numerical analysis of the form factor, field map, and field distribution 
is the subject of Sec. VII. We end up with a discussion and the conclusions in Sec. VIII. Analytical and numerical details can be found in five Appendices. In particular, the last appendix shows that the form factor can be expressed in terms of a reduced number of parameters. This result allows in Sec. VII easily studying the crossover from BCS to GL in the VL structures.

\section{GEOMETRY}

We assume $\mathbf{B}_{\text {ext }}$ to be applied along the $Z$ axis of an orthogonal reference frame, with the vortex tubes of a type-II superconductor in its mixed phase running along that axis. The VL is taken to be composed of equilateral triangles. Therefore in the direct space the unit cell is defined by the three vectors $\mathbf{v}_{1}=X_{1} \hat{\mathbf{x}}, \mathbf{v}_{2}=X_{2} \hat{\mathbf{x}}+Y_{2} \hat{\mathbf{y}}$, and $\mathbf{v}_{3}=\hat{\mathbf{z}}$, where $\hat{\mathbf{x}}$ and $\hat{\mathbf{y}}$ are two mutually orthogonal unit vectors perpendicular to the $Z$ axis, $\hat{\mathbf{z}}$ is the unit vector of the $Z$ axis, and $\left\{X_{1}, X_{2}, Y_{2}\right\}$ are coordinates with the relations $X_{2}=X_{1} / 2$ and $Y_{2}=\sqrt{3} X_{1} / 2$. Since the vortex tubes are taken as straight, a VL point is labeled by the two-dimensional vector

$$
\mathbf{R}_{p, q}=p \mathbf{v}_{1}+q \mathbf{v}_{2}=\left(p X_{1}+q X_{2}\right) \hat{\mathbf{x}}+q Y_{2} \hat{\mathbf{y}} .
$$

Following Delrieu's convention a lattice point in the reciprocal lattice is specified by the reciprocal vector

$$
\mathbf{K}_{m, h}=-h \mathbf{v}_{1}^{*}+m \mathbf{v}_{2}^{*}=\frac{2 \pi}{s_{\mathrm{c}}}\left[-h Y_{2} \hat{\mathbf{x}}+\left(m X_{1}+h X_{2}\right) \hat{\mathbf{y}}\right],
$$

with the VL unit cell area

$$
s_{\mathrm{c}}=X_{1} Y_{2}=\frac{\Phi_{0}}{\overline{B^{Z}}} .
$$

The mean value of the induction is denoted $\overline{B^{Z}(\mathbf{r})}$ or $\overline{B^{Z}}$ in short, where $\mathbf{r}$ refers to a position in the direct space. We have introduced the magnetic flux quantum $\Phi_{0}\left(\Phi_{0}=2.06783 \times\right.$ $10^{-15} \mathrm{~T} \mathrm{~m}^{2}$ ), and the two vectors $\mathbf{v}_{1}^{*}$ and $\mathbf{v}_{2}^{*}$ which define the unit cell in the reciprocal lattice.

As examples, with an obvious notation, we have $\quad \mathbf{R}_{1,0}=X_{1}(1,0), \quad \mathbf{R}_{0,1}=X_{1}(1 / 2, \sqrt{3} / 2), \quad$ and $\mathbf{R}_{-1,1}=X_{1}(-1 / 2, \sqrt{3} / 2)$. For the reciprocal lattice points, we compute $\mathbf{K}_{1,0}=\left[4 \pi /\left(\sqrt{3} X_{1}\right)\right](0,1)$, $\mathbf{K}_{0,1}=\left[4 \pi /\left(\sqrt{3} X_{1}\right)\right](-\sqrt{3} / 2,1 / 2), \quad$ and $\quad \mathbf{K}_{-1,1}=$ $\left[4 \pi /\left(\sqrt{3} X_{1}\right)\right](-\sqrt{3} / 2,-1 / 2)$. The complementary three points in the direct and reciprocal lattices respectively can be obtained by symmetry.

We introduce for convenience two reduced quantities. We define the magnetic length

$$
\Lambda=\sqrt{\frac{s_{\mathrm{c}}}{2 \pi}}=\sqrt{\frac{X_{1} Y_{2}}{2 \pi}}=\sqrt{\frac{\Phi_{0}}{2 \pi \overline{B^{Z}}}} .
$$

It is related to the lattice parameter of the VL:

$$
X_{1}=\left(\frac{4}{3}\right)^{1 / 4} \sqrt{\frac{\Phi_{0}}{\overline{B^{Z}}}}=2.693 \times \Lambda .
$$

Unless $B_{\text {ext }}$ is close to the lower critical field $B_{\mathrm{c} 1}$, the magnetization of a superconductor is negligible, and therefore $\overline{B^{Z}} \simeq B_{\text {ext }}$. We shall express the form factor with the unitless parameter $n_{\mathbf{K}_{m, h}}$ defined through the wave-vector scalar product

$$
\mathbf{K}_{m, h} \cdot \mathbf{K}_{m, h}=K_{m, h}^{2}=\frac{4}{\Lambda^{2}} n_{\mathbf{K}_{m, h}}^{2} .
$$

From this definition, we derive the important formula

$$
n_{\mathbf{K}_{m, h}}^{2}=\frac{\pi X_{1}}{2 Y_{2}}\left(m^{2}+m h+h^{2}\right)=\frac{\pi}{\sqrt{3}}\left(m^{2}+m h+h^{2}\right) .
$$

\section{PHYSICAL PRINCIPLES FOR THE DESCRIPTION OF THE MAGNETIZATION AND FORM FACTOR}

The computations of the magnetization and form factor are based on an approximate form of the integral Gorkov equation for the temperature Green's function $G_{\omega_{\ell}}\left(\mathbf{r}, \mathbf{r}^{\prime}\right):{ }^{16}$

$$
\begin{aligned}
G_{\omega_{\ell}}\left(\mathbf{r}, \mathbf{r}^{\prime}\right)= & G_{\omega_{\ell}}^{n}\left(\mathbf{r}-\mathbf{r}^{\prime}\right)-\int G_{\omega_{\ell}}^{n}\left(\mathbf{r}-\mathbf{r}_{1}\right) V\left(\mathbf{r}_{1}, \mathbf{r}_{2}\right) \\
& \times G_{-\omega_{\ell}}^{n}\left(\mathbf{r}_{2}-\mathbf{r}_{1}\right) G_{\omega_{\ell}}\left(\mathbf{r}_{2}, \mathbf{r}^{\prime}\right) d^{3} \mathbf{r}_{1} d^{3} \mathbf{r}_{2},
\end{aligned}
$$

where $V\left(\mathbf{r}_{1}, \mathbf{r}_{2}\right)$ is the correlation function of the order parameter $\Delta(\mathbf{r})$ :

$$
V\left(\mathbf{r}_{1}, \mathbf{r}_{2}\right)=\Delta\left(\mathbf{r}_{1}\right) \Delta^{*}\left(\mathbf{r}_{2}\right) \exp \left(-\frac{2 i e}{h} \int_{\mathbf{r}_{1}}^{\mathbf{r}_{2}} \mathbf{A}(\mathbf{l}) \cdot d \mathbf{l}\right) .
$$

Note that $V\left(\mathbf{r}_{1}, \mathbf{r}_{2}\right)$ describes the correlation function of the Cooper pairs rather than the correlation of the electrons. A justification of the correlation function nature of $V\left(\mathbf{r}_{1}, \mathbf{r}_{2}\right)$ is given after Eq. (13). We have introduced the Matsubara angular precession frequency $\omega_{\ell}$ :

$$
\omega_{\ell}=(2 \ell+1) \pi k_{\mathrm{B}} T / \hbar .
$$

The path of integration over the potential vector $\mathbf{A}$ is a straight line between $\mathbf{r}_{1}$ and $\mathbf{r}_{2}$. Within the semiclassical approximation the effect of the field on the material is entirely described by the phase integral in the correlation function and $G_{\omega_{\ell}}^{n}\left(\mathbf{r}-\mathbf{r}^{\prime}\right)$ refers to the temperature Green's function of the normal metal in the absence of a magnetic field. The semiclassical approximation is valid if the spacing between the Landau levels is small compared to the sum of their thermal and collision broadenings; i.e., ${ }^{16}$

$$
\mathcal{R}_{\phi}=\frac{\mu_{\mathrm{B}} B_{\text {ext }}}{2 \pi k_{\mathrm{B}} T+\hbar / \tau_{\text {life }}} \ll 1 .
$$

Here $\tau_{\text {life }}$ is the level lifetime which accounts for the finite electron mean-free path. It is further discussed in Sec. VIII. Neglecting the $\hbar / \tau_{\text {life }}$ term in the ratio expression, we compute $\mathcal{R}_{\phi}=1$ when $T=0.04 \mathrm{~K}$ for $B_{\text {ext }}=0.4 \mathrm{~T}$. This $B_{\text {ext }}$ value corresponds approximately to $B_{\mathrm{c} 2}$ for a high-quality niobium sample. ${ }^{14}$ The theory for the form factor and resulting field map and field distribution discussed here is therefore expected to be valid down to $0.04 \mathrm{~K}$ for simple superconductors such as niobium when the VL is composed of equilateral triangles.

The correlation function has the periodicity of the VL with respect to the center of mass of a Cooper pair, i.e., $\left(\mathbf{r}_{1}+\right.$ $\left.\mathbf{r}_{2}\right) / 2 .{ }^{11}$ Therefore it can be Fourier expanded. Neglecting the spatial variation of the induction, a valid approximation in our case since we are interested in the high-field VL for which the 
magnetization is negligible, ${ }^{4,12}$

$$
V\left(\mathbf{r}_{1}, \mathbf{r}_{2}\right)=\sum_{m, h} V_{\mathbf{K}_{m, h}}\left(\mathbf{r}_{1}-\mathbf{r}_{2}\right) \exp \left[i \mathbf{K}_{m, h} \cdot\left(\mathbf{r}_{1}+\mathbf{r}_{2}\right) / 2\right],
$$

with

$$
V_{\mathbf{K}_{m, h}}(\mathbf{r})=(-1)^{m h} V_{0}\left(\mathbf{r}-\mathbf{R}_{m, h}\right) .
$$

Since $V\left(\mathbf{r}_{1}, \mathbf{r}_{2}\right)$ depends on the difference $\left(\mathbf{r}_{1}-\mathbf{r}_{2}\right)$ and the center of mass of the Cooper pairs, and not on $\mathbf{r}_{1}$ and $\mathbf{r}_{2}$ individually, it is really a correlation function. The Fourier components have a remarkable property: $V_{\mathbf{K}_{m, h}}\left(\mathbf{R}_{m, h}\right)=$ $(-1)^{m h} V_{0}(0)$. Therefore at the VL nodes, i.e., at position $\mathbf{R}_{m, h}$, within a phase the correlation function of the order parameter has a common single value. In fact the phase changes from one position to the next-nearest neighbors in a coherent fashion. Because of this long-range coherence, we expect Cooper pair diffraction on the vortex cores. However, the diffraction can only be partial since the coherent nature of the correlation is only active for a finite set of Cooper pairs, i.e., the ballistic ones with trajectory through the vortex cores. It is the extended correlation which is the origin of the slow power decay of the form factor at low temperature discussed latter on. This property does not hold near $B_{\mathrm{c} 1}$ since the spatial variation of the induction has been neglected in deriving Eq. (13).

As the correlation function, the Green's function is periodic with respect to $\left(\mathbf{r}_{1}+\mathbf{r}_{2}\right) / 2$. In addition to the half sum of the coordinates, the two functions depend on the difference of the coordinates. To the difference corresponds the continuous conjugate Fourier vector $\mathbf{p}$. Performing the $\mathbf{K}_{m, h}$ and $\mathbf{p}$ Fourier transforms on the approximate Gorkov equation given at Eq. (8), an algebraic set of equations is derived. An approximate simple solution has been proposed in Ref. 10 based on the fact that the terms of the set with $\mathbf{K}_{m, h} \neq \mathbf{0}$ are negligible relative to the ones with $\mathbf{K}_{m, h}=\mathbf{0}$. Latter on, it has been shown to be satisfactory only for the computation of thermodynamic quantities. It cannot be used to describe the dynamics. It is valid even at low temperature. ${ }^{12}$

The free energy of a superconductor can be written in terms of $G_{\omega_{\ell}}\left(\mathbf{r}, \mathbf{r}^{\prime}\right) .{ }^{17}$ Focusing again on the case for which $B_{\text {ext }}$ is near $B_{\mathrm{c} 2}$, and therefore the spatial variation of the order parameter can be neglected, and assuming the Abrikosov vortex solution, ${ }^{4,11}$

$$
\begin{aligned}
F= & \Delta_{0}^{2} N_{0} \ln \left(\frac{T}{T_{\mathrm{c} 0}}\right)-8 \pi k_{\mathrm{B}} T N_{0} \\
& \times \sum_{\ell=0}^{\infty}\left[\int_{0}^{\pi / 2} \sin \theta\left(\frac{u_{\ell}}{2 a}-\hbar \omega_{\ell}\right) d \theta-\frac{\Delta_{0}^{2}}{4 \hbar\left|\omega_{\ell}\right|}\right] .
\end{aligned}
$$

We have defined the function

$$
a=a(\theta)=\frac{\Lambda}{\hbar v_{\mathrm{F}} \sin \theta}
$$

and the variable $u_{\ell}$ which is the root of the equation

$$
u_{\ell}=2 \hbar \omega_{\ell} a+\Delta_{0}^{2} a^{2} i v\left(i u_{\ell}\right) \text {. }
$$

The Fermi surface has been assumed to be spherical. As we are using the BCS theory, we are working in the weak-coupling limit. The function $v(z)$ is defined in Appendix A.
In addition to the temperature, the free energy depends on four parameters: the critical temperature at low field $T_{\mathrm{c} 0}$, the mean order parameter squared $\Delta_{0}^{2}=\overline{|\Delta(\mathbf{r})|^{2}}$ where the bar is for the spatial averaging, the density of states at the Fermi energy per spin, volume, and energy in the normal state $N_{0}$, and the Fermi velocity $v_{\mathrm{F}}$. The order parameter can be related to basic parameters of the superconductor. Assuming that the average value $\Delta_{0}$ is equal to the value of the order parameter in zero field, according to BCS

$$
\frac{\Delta_{0}(0)}{k_{\mathrm{B}} T_{\mathrm{c} 0}}=\frac{\pi}{\exp (\gamma)}=1.7639,
$$

where $\Delta_{0}(0)$ is the value of $\Delta_{0}$ at $T=0$ and $\gamma$ the EulerMascheroni constant; i.e., $\gamma=0.57722$. So at low temperature only three material parameters are left if the spherical Fermi surface and weak-coupling approximations are valid.

In addition to $\Delta_{0}(0)$, other parameters characterize a superconductor. The Pippard-BCS coherence length $\xi_{0}(0)$ is related to $\Delta_{0}(0)$,

$$
\xi_{0}(0)=\frac{\hbar v_{\mathrm{F}}}{\pi \Delta_{0}(0)},
$$

and in the clean limit (see for example Ref. 1 at page 120),

$$
\xi_{\mathrm{GL}}=\xi_{0} / 0.96 \text {. }
$$

Here $\xi_{\mathrm{GL}}$ is the GL coherence length. We stress that Eq. (19) is derived in the $T=0$ limit. We note the GL relation

$$
B_{\mathrm{c} 2}(T)=\frac{\Phi_{0}}{2 \pi \xi_{\mathrm{GL}}^{2}(T)} .
$$

It is also possible to derive information on the mean order parameter from the minimization of the free energy written above. This leads to a formula of the Helfand-Werthamer type $^{18}$ which can be used to model $B_{\mathrm{c} 2}(T)$ for an isotropic Fermi surface in the weak-coupling approximation. However, that type of formula does not describe $B_{\mathrm{c} 2}(T)$ for niobium, ${ }^{19,20}$ one of the metals for which the theory discussed here may apply. This is attributed to the strong anisotropy of the Fermi surface. ${ }^{21,22}$ Hence, we shall not pursue any longer our discussion of $\Delta_{0}$ in terms of the free energy discussed in this work.

\section{MAGNETIZATION}

While our main purpose in this report is the study of the form factor, here we discuss the magnetization $M$. This is justified since, thanks to Abrikosov, the relation between $M$ and the induction is known for a temperature in the vicinity of $T_{\mathrm{c} 0}$. Therefore the study of $M$ gives us the possibility to check the validity of the formula for the induction, and therefore the form factor.

We recall that $M=-\partial F / \partial \overline{B^{Z}}$. In terms of the free-energy expression given by Eq. (14), $\partial F / \partial \overline{B^{Z}}=$ $-\left[a /\left(2 \overrightarrow{B^{Z}}\right)\right](\partial F / \partial a)$ and therefore we need to evaluate $a(\partial / \partial a)\left(u_{\ell} / a\right)$. This is done in Appendix B. We finally obtain

$$
M=\frac{\pi k_{\mathrm{B}} T N_{0} \Delta_{0}^{2}}{2 \overline{B^{Z}}} \sum_{\ell=0}^{\infty} \int_{0}^{\pi / 2} \sin (\theta) i v^{\prime \prime}\left(i u_{\ell}\right) \frac{\partial u_{\ell}}{\partial\left(\hbar \omega_{\ell}\right)} d \theta .
$$

$M$ can be evaluated numerically and compared to experimental data. We shall use it in our discussion of the form factor. 
Interestingly, it can be drastically simplified in the $T=0$ limit. From Appendix C

$$
M=-\frac{N_{0} \Delta_{0}^{2}}{2 \overline{B^{Z}}} .
$$

This result will be used in Sec. V. As expected, $M$ is negative.

\section{FORM FACTOR}

Here we consider the field Fourier component $B_{\mathbf{K}_{m, h}}^{Z}$ which is usually called the form factor in the SANS literature. It is related to the space-dependent induction component $B^{Z}(\mathbf{r})$ by the Fourier relation

$$
B^{Z}(\mathbf{r})=\sum_{\mathbf{K}_{m, h}} B_{\mathbf{K}_{m, h}}^{Z} \exp \left(i \mathbf{K}_{m, h} \cdot \mathbf{r}\right),
$$

with

$$
B_{\mathbf{K}_{m, h}}^{Z}=\frac{1}{s_{\mathrm{c}}} \int_{s_{\mathrm{c}}} B^{Z}(\mathbf{r}) \exp \left(-i \mathbf{K}_{m, h} \cdot \mathbf{r}\right) d^{2} \mathbf{r} .
$$

According to Refs. 4 and 12 and correcting for misprints,

$$
\begin{aligned}
B_{\mathbf{K}_{m, h}}^{Z}= & -\frac{\mu_{0} \pi N_{0} \Delta_{0}^{2}}{\overline{B^{Z}}} \frac{(-1)^{m h} \exp \left(-n_{\mathbf{K}_{m, h}}^{2}\right)}{n_{\mathbf{K}_{m, h}}^{2}} 2 \pi k_{\mathrm{B}} T \\
& \times \sum_{\ell=0}^{\infty} \int_{0}^{\pi / 2} \sin (\theta) g_{\ell}(\theta) d \theta,
\end{aligned}
$$

where we have introduced the auxiliary function

$$
\begin{aligned}
g_{\ell}(\theta)= & \frac{i v\left(i u_{\ell}+i n_{\mathbf{K}_{m, h}}\right)+i v\left(i u_{\ell}-i n_{\mathbf{K}_{m, h}}\right)-2 i v\left(i u_{\ell}\right)}{\left[1+\frac{\Delta_{0}^{2} \Lambda^{2}}{\hbar^{2} v_{\mathrm{F}}^{2} \sin ^{2} \theta} v^{\prime}\left(i u_{\ell}\right)\right]^{2}} \\
& \times \frac{\partial u_{\ell}}{\partial\left(\hbar \omega_{\ell}\right)} .
\end{aligned}
$$

It is convenient to rewrite the variable $u_{\ell}$ without the intermediate function $a(\theta)$. Referring to Eqs. (15) and (16),

$$
u_{\ell}=2 \omega_{\ell} \frac{\Lambda}{v_{\mathrm{F}} \sin \theta}+\frac{\Delta_{0}^{2} \Lambda^{2}}{\hbar^{2} v_{\mathrm{F}}^{2} \sin ^{2} \theta} i v\left(i u_{\ell}\right) .
$$

Therefore $g_{\ell}(\theta)$ is indeed a function of the angle $\theta$ and the angular frequency $\omega_{\ell}$.

This formula for $B_{\mathbf{K}_{m, h}}^{Z}$ is extremely complicated. Remarkably, as $M$ does, $B_{\mathbf{K}_{m, h}}^{Z}$ depends on three material parameters, i.e., $\Delta_{0}^{2}, N_{0}$, and $v_{\mathrm{F}}$, and $T$ and $\bar{B}^{Z}$. This suggests that simple relations may exist between them. They do exist in two limiting cases. Interestingly, $N_{0}$ only appears as a proportionality parameter.

In the proximity of $T_{\mathrm{c} 0}$ the asymptotic limit of the form factor is (see Appendix D 1)

$$
B_{\mathbf{K}_{m, h}}^{Z}=\mu_{0} M(-1)^{m h} \exp \left(-n_{\mathbf{K}_{m, h}}^{2}\right) .
$$

Recalling the definition of the Fourier transform of the induction, see Eq. (23),

$$
B^{Z}(\mathbf{r})=\overline{B^{Z}}+\sum_{(m, h) \neq(0,0)} B_{\mathbf{K}_{m, h}}^{Z} \exp \left(i \mathbf{K}_{m, h} \cdot \mathbf{r}\right) .
$$

This means that

$$
\begin{aligned}
B^{Z}(\mathbf{r})= & \overline{B^{Z}}-\mu_{0}|M| \sum_{(m, h) \neq(0,0)}(-1)^{m h} \exp \left(-n_{\mathbf{K}_{m, h}}^{2}\right) \\
& \times \exp \left(i \mathbf{K}_{m, h} \cdot \mathbf{r}\right),
\end{aligned}
$$

since $M<0$; see Sec. IV. This is the Abrikosov result. ${ }^{23}$

An exotic behavior is found in the $T \rightarrow 0$ limit. From Appendix D 2 for $n_{\mathbf{K}_{m, h}} \gg 1$,

$$
B_{\mathbf{K}_{m, h}}^{Z}=-\frac{\mu_{0} \pi \sqrt{\pi} N_{0} \Delta_{0}^{2}}{\overline{B^{Z}}} \frac{(-1)^{m h}}{n_{\mathbf{K}_{m, h}}^{3}} .
$$

In terms of the magnetization, see Eq. (22), it gives

$$
B_{\mathbf{K}_{m, h}}^{Z}=-2 \pi \sqrt{\pi} \mu_{0}|M| \frac{(-1)^{m h}}{n_{\mathbf{K}_{m, h}}^{3}} .
$$

Whereas $B_{\mathbf{K}_{m, h}}^{Z}$ has a Gaussian wave-vector dependence near $T_{\mathrm{c} 0}$, it decays much more slowly with a wave-vector power law at low temperature. This is quantified in Sec. VII. Hence, whereas only the low-indices Bragg spots might be observed by small-angle neutron scattering (SANS) near $T_{\mathrm{c} 0}$, more spots should be detected at low temperature. In fact, in addition to the usual six $\mathbf{K}_{1,0}$ Bragg reflections, $\mathbf{K}_{1,1}$ and $\mathbf{K}_{2,0}$ spots have already been observed for niobium at $4.2 \mathrm{~K}$ for $\mathbf{B}_{\text {ext }}$ parallel to the [111] crystal direction. ${ }^{24}$ Only for that field direction is the VL triangular, and hence is of interest here. Because of possible double Bragg scattering effects and large distortions in the VL, it is not easy to derive information from the published form-factor data on niobium to compare with the power-law prediction.

\section{FIELD DISTRIBUTION}

It is difficult to analyze analytically the spatial dependence of the induction. Here we shall focus on the field distribution as measured by $\mu \mathrm{SR}$ and NMR techniques. We shall assume a disorder-free VL and the field width of the distribution to be small relative to $\overline{B^{Z}}$; i.e., only the distribution of the field component along $\mathbf{B}_{\text {ext }}$ is measured. ${ }^{25}$ This latter condition can be checked to be fulfilled by looking at the computed distribution. For simplicity we shall write the distribution $D_{\mathrm{c}}\left(B^{Z}\right)$ without specifying that it does depend on $B_{\text {ext }}$.

Mathematically, the distribution can be expressed in terms of a two-dimensional Dirac function:

$$
D_{\mathrm{c}}\left(B^{Z}\right)=\int_{s_{\mathrm{c}}} \delta\left[B^{Z}(\mathbf{r})-B^{Z}\right] \frac{d^{2} \mathbf{r}}{s_{\mathrm{c}}},
$$

where the integral only extends over the unit cell. An important characterization of a distribution is its variance: ${ }^{25}$

$$
\Delta_{Z, \mathbf{v}}^{2}=\sum_{\mathbf{K} \neq \mathbf{0}}\left|B_{\mathbf{K}}^{Z}\right|^{2} \text {. }
$$

For the simple asymptotic form factor given in Eq. (31), we derive for the standard deviation

$$
\begin{aligned}
\Delta_{Z, \mathrm{v}} & =\frac{3^{3 / 4} \mu_{0} N_{0} \Delta_{0}^{2}}{\overline{B^{Z}}} \sqrt{\sum_{(m, h) \neq(0,0)} \frac{1}{\left(m^{2}+m h+h^{2}\right)^{3}}} \\
& =5.756 \frac{\mu_{0} N_{0} \Delta_{0}^{2}}{\overline{B^{Z}}} .
\end{aligned}
$$




\section{NUMERICAL ANALYSIS OF THE FORM FACTOR, FIELD MAP, AND FIELD DISTRIBUTION}

As shown in Appendix E 1, the form factor $B_{\mathbf{K}_{m, h}}^{Z}$ depends in fact only on three parameters noted $\tilde{a}, \tilde{b}$, and $\tilde{c}$. The parameter $\tilde{a}=-\mu_{0} \pi N_{0} \Delta_{0}^{2} 2 \tilde{c} / \overline{B^{Z}}$ stands as a proportionality coefficient while $\tilde{b}$ and $\tilde{c}$ are dimensionless $\left[\tilde{b}=\left(\Lambda / \pi \xi^{B}\right)^{2}\right.$ and $\tilde{c}=\Lambda / \xi^{T}$ with the temperature and field dependent length scale parameters $\xi^{T}=\hbar v_{\mathrm{F}} /\left(2 \pi k_{\mathrm{B}} T\right)$ and $\xi^{B}=\hbar v_{\mathrm{F}} / \pi \Delta_{0}$; see Appendix E 1]. Interestingly, for $B_{\mathrm{ext}} \simeq B_{\mathrm{c} 2} \tilde{b} \ll 1$ and $\tilde{c}$ changes notably as $T$ varies. As shown below, this enables us to easily discuss the physics of the form factor, field map, and field distribution.

Let us consider a classical BCS superconductor with $T_{\mathrm{c} 0}=10 \mathrm{~K}$. From Eq. (17) we compute $\Delta_{0}(0) \simeq 1.52 \mathrm{meV}$. Assuming $B_{\mathrm{c} 2}(0)=0.4 \mathrm{~T}$, from the GL relation [Eq. (20)] we find $\xi_{\mathrm{GL}}(0)=28.7 \mathrm{~nm}$. Using the BCS relation $v_{\mathrm{F}}=$ $\xi_{0} \pi \Delta_{0}(0) / \hbar$, see Eq. (18), and the approximation of $\xi_{0}$ in terms of $\xi_{\mathrm{GL}}$ given at Eq. (19), we estimate $v_{\mathrm{F}} \simeq 2.00$ $\times 10^{5} \mathrm{~m} / \mathrm{s}$. Let us assume for the second critical field the simple temperature dependence $B_{\mathrm{c} 2}(T)=B_{\mathrm{c} 2}(0)\left(1-t^{2}\right)$, with the reduced temperature $t=T / T_{\mathrm{c} 0}$. The field and temperature dependencies of the gap are taken to be described by the compact formula $\Delta_{0}=\Delta_{0}(T) \sqrt{1-b}=\Delta_{0}(0) \sqrt{1-b} \sqrt{1-t^{2}}$, with the reduced field $b=B_{\text {ext }} / B_{\mathrm{c} 2}(T)$. Here we note that $\xi_{0} \neq \xi^{B}$ since $\xi_{0}$ only depends on $\Delta_{0}(0)$ while $\xi^{B}$ is a function of $\Delta_{0}$. Under these assumptions,

$$
\begin{aligned}
\tilde{b} & =\left(\frac{\Lambda \Delta_{0}}{\hbar v_{\mathrm{F}}}\right)^{2}=\frac{\Delta_{0}^{2}(T)(1-b)}{\hbar^{2} v_{\mathrm{F}}^{2}} \frac{\Phi_{0}}{2 \pi B_{\mathrm{c} 2}(T)} \frac{B_{\mathrm{c} 2}(T)}{\overline{B^{Z}}} \\
& =\frac{1}{\pi^{2}} \frac{\xi_{\mathrm{GL}}^{2}(T)}{\xi_{0}^{2}(T)} \frac{1-b}{b} \simeq 0.110 \frac{1-b}{b} .
\end{aligned}
$$

Above we used Eqs. (18)-(20). The parameter $\tilde{c}$ is field and temperature dependent:

$$
\tilde{c}=1.1811 \frac{t}{\sqrt{b\left(1-t^{2}\right)}} .
$$

Interestingly, $\tilde{c}$ is only written in terms of two reduced variables. Numerically, $\tilde{b}=0.110,0.073,0.047,0.027,0.012$, and 0.0011 for $b=0.5,0.6,0.7,0.8,0.9$, and 0.99 , respectively. $\tilde{c}$ is linear in temperature in the low-temperature limit and diverges as $t \rightarrow 1$. The temperature dependence of $\tilde{c}$ for $b=0.5,0.7,0.9$, and 0.99 is given in Fig. 1. In agreement with our discussion in Appendix E1, while the $\tilde{c}$ thermal dependence is quite strong and it gets large as the critical temperature is approached, $\tilde{b}$ is weakly temperature dependent and has a negligibly small value in the $B_{\mathrm{ext}} \rightarrow B_{\mathrm{c} 2}$ limit.

The analysis of $\tilde{b}$ and $\tilde{c}$ suggests to start our discussion of the VL properties focusing on its $\tilde{c}$ dependence near the $\tilde{b} \rightarrow 0$ limit. In Figs. 2 and 3 we show the contour plots of the spatial field distribution for $\tilde{b}=0.0011$ (i.e., $b=0.99$ ) and $\tilde{c}=0.01,0.10,0.30,0.70,0.90,1.30,3.00$, and 20.00. Comparing the data at the top of Fig. 2 and bottom of Fig. 3, the field map, profile, field distribution, and form factor intensity are drastically different at low temperature and near $T_{\mathrm{c} 0}$. At $\tilde{c}=$ $\tilde{c}_{\text {cross }} \simeq 0.9$ a crossover occurs. This value of $\tilde{c}_{\text {cross }}$ corresponds to $T \simeq 0.6 T_{\mathrm{c} 0}$ in agreement with a report of $\mathrm{E}$. $\mathrm{H}$. Brandt (this value depends on $\left.v_{\mathrm{F}}\right){ }^{26}$

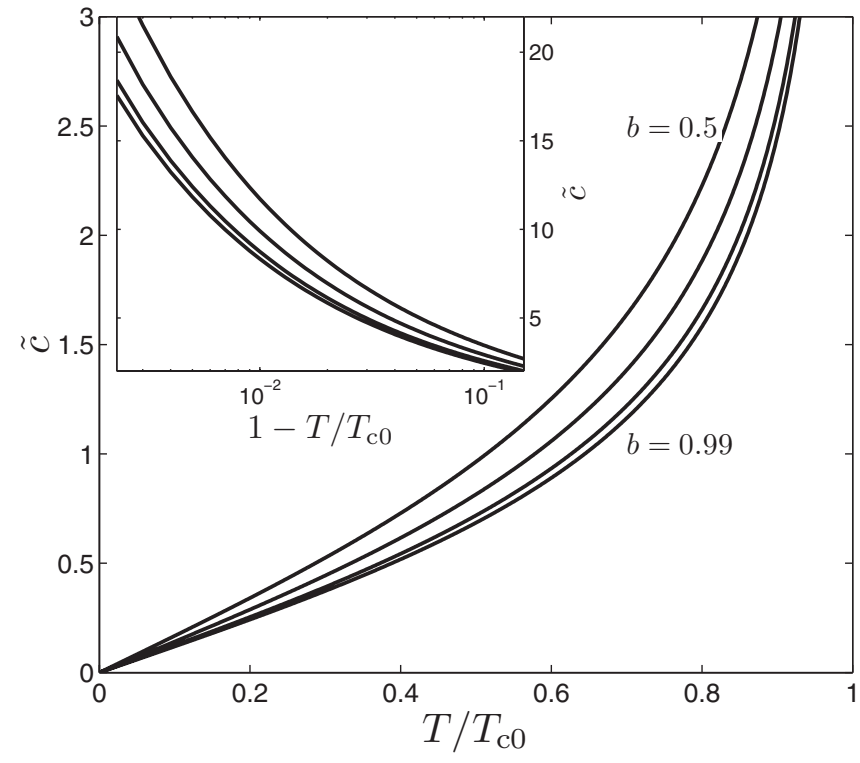

FIG. 1. Temperature dependence of the parameter $\tilde{c}$ in reduced temperature scale for four different values of the reduced field $b=$ $B_{\text {ext }} / B_{\text {c2 }}$, i.e., $b=0.5,0.7,0.9$, and 0.99 . The insert shows the same but on semilogarithmic scale in the vicinity of $T_{\mathrm{c} 0}$.

For $\tilde{c}>\tilde{c}_{\text {cross }}$ we do observe behaviors expected in the GL regime. ${ }^{25,27} \mathrm{~A}$ minimum for the spatial field distribution is found in between three neighboring vortices, while in between two neighboring vortices, i.e., at midpoint on the line connecting two neighboring vortices, a field saddle point is located. The signature of the minimum of the field is obvious in $D_{\mathrm{c}}\left(B^{Z}\right)$. The saddle point corresponds to the maximum in $D_{\mathrm{c}}\left(B^{Z}\right)$. As shown in the inserts of the field distributions, the intensity of the form factor is expected to be reduced for large indices.

For $\tilde{c}<\tilde{c}_{\text {cross }}$ the positions of the minimum and saddle point are reversed. The shape of the profiles are substantially different. In the limit $\tilde{c} \rightarrow 0$ the conical shape of the vortex profiles near the positions of the minimum and saddle point are particularly pronounced. This property is even more clearly seen at the minimum-field point as shown by the dashed line in the field profile. This feature reflects directly the weak power-law decay of the form factor which is a consequence of the Cooper pair diffraction by the vortex cores. This can be qualitatively understood if we recall that the weight of the $k$ harmonic for the infinite Fourier series of a triangle wave-a wave with a profile similar to the dashed line-is proportional to $(-1)^{k} /(2 k+1)^{2}$. Note the alternating sign as in the $B_{\mathbf{K}_{m, h}}^{Z}$ expression in the $T=0$ limit, as well as the power-law decay. As reflected by the different exponents of the power-law decays, this comparison is only qualitative. This is not surprising given the two-dimensional nature of the field map. The triangle-wave model has only one dimension. For $\tilde{c} \geqslant 0.70$ the BCS solution and the GL limit, i.e., $T \rightarrow T_{\mathrm{c} 0}$, predict similar high-field $D_{\mathrm{c}}\left(B^{Z}\right)$. But the low-field features are still different. As noticed from Fig. 2, it is not required to go to extremely low temperature to observe Bragg's spots of large indices when $B_{\mathrm{ext}} \rightarrow B_{\mathrm{c} 2}$.

A linear high-field tail is predicted in Fig. 2 for $D_{\mathrm{c}}\left(B^{Z}\right)$. However, it is only expected at really low temperature, i.e., for 

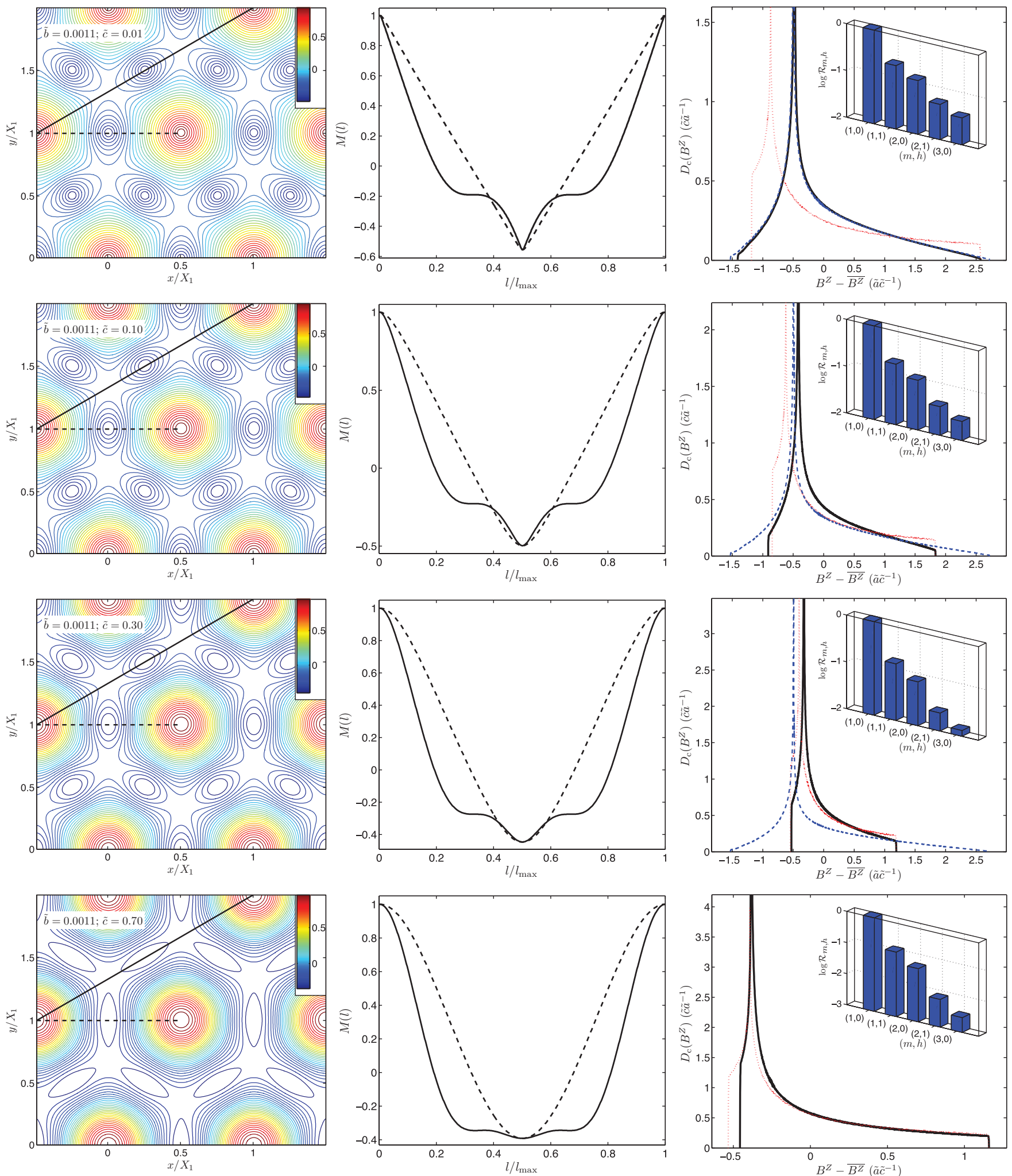

FIG. 2. (Color online) Left panels: Contour plot $M(x, y)=\left[B^{Z}(x, y)-\overline{B^{Z}}\right] /\left[B_{\mathrm{vc}}^{Z}-\overline{B^{Z}}\right]$ for $\tilde{b}=0.0011$ and four different $\tilde{c}$ values, i.e., $\tilde{c}=0.01,0.10,0.30$, and 0.70, from top to bottom. Middle panels: Field profile along the solid and the dashed lines shown in the left panels. Right panels: The corresponding component field distribution $D_{\mathrm{c}}\left(B^{Z}\right)$ is shown as a solid black line. For comparison, we present in dashed blue and dotted red lines $D_{\mathrm{c}}\left(B^{Z}\right)$ for $\tilde{c} \rightarrow 0$ and $\tilde{c} \rightarrow \infty$, respectively. In order to match the vortex core field $B_{\mathrm{vc}}^{Z}$, the horizontal axis for $D_{\mathrm{c}}\left(B^{Z}\right)$ shown with the dotted red line $(\tilde{c} \rightarrow \infty)$ has been scaled $\left(\overline{B^{Z}}\right.$ is identical for all the curves). The insert shows the values of $\mathcal{R}_{m, h}=\left|B_{\mathbf{K}_{m, h}}^{Z} / B_{\mathbf{K}_{10}}^{Z}\right|$ for the first five form factors in logarithm scale. To simplify the drawings, and without any loss of information, the dashed blue line for the $D_{\mathrm{c}}\left(B^{Z}\right)$ plots (corresponding to $\tilde{c} \rightarrow 0$ ) is presented only when $\tilde{c} \leqslant 0.30$. 

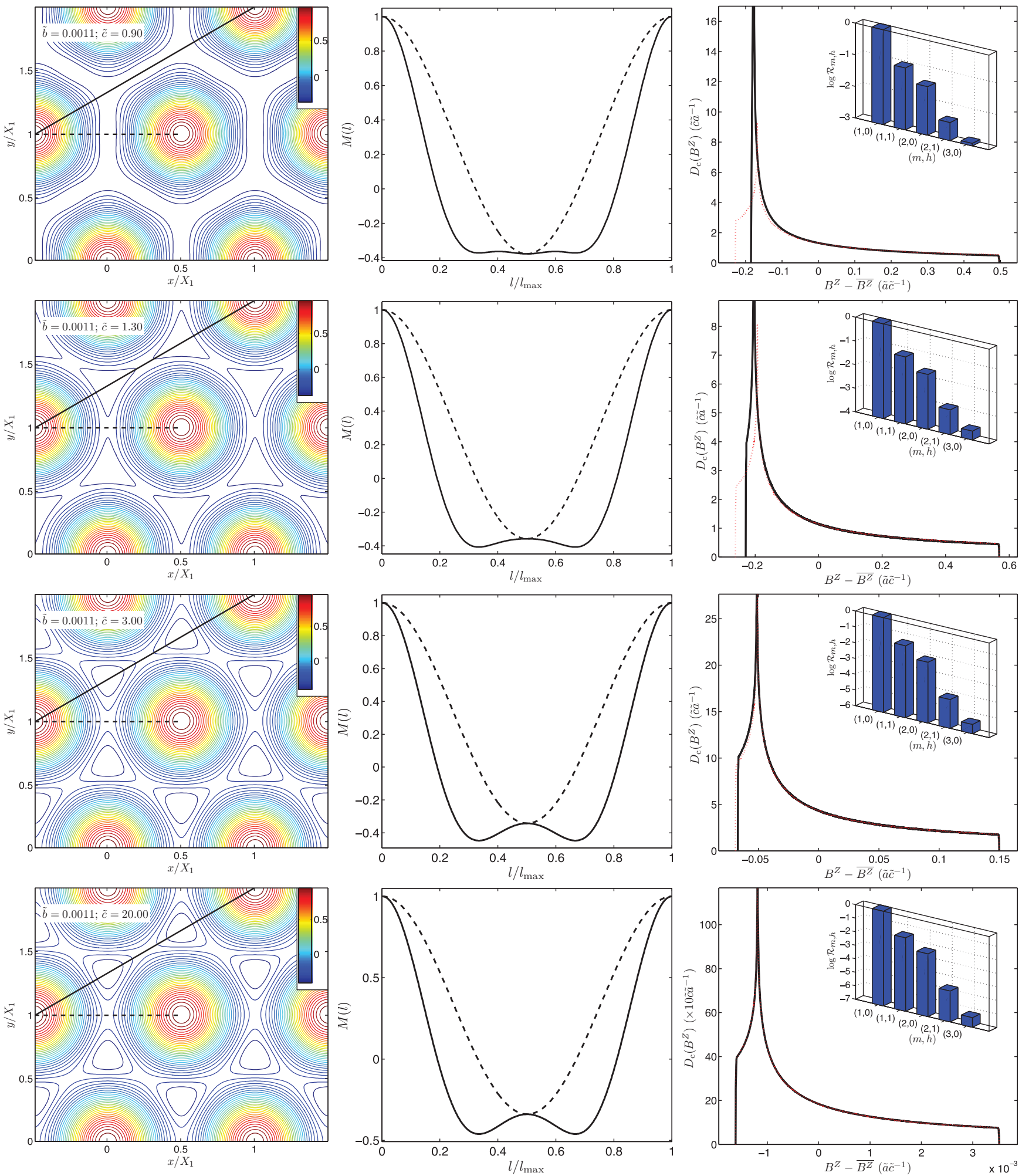

FIG. 3. (Color online) The same as in Fig. 2, but for $\tilde{c}=0.90,1.30$, 3.00, and 20.00, from top to bottom, respectively.

$\tilde{c} \leqslant 0.10$, in contrast to observation. ${ }^{14}$ The $D_{\mathrm{c}}\left(B^{Z}\right)$ shape near the minimum field is really different near $T=0$ and $T_{\mathrm{c} 0}$. This difference remains to be seen experimentally. A signature of the crossover for $D_{\mathrm{c}}\left(B^{Z}\right)$ is in its sharp rise at low field without any shoulder. Although never reported, it could be observed experimentally.
Up to now we have focused our attention on the physics very near the $B_{\mathrm{c} 2}$ phase boundary when the parameter $\tilde{b}$ is negligible. Now we study it as we go out of the boundary. In Fig. 4 we present the maps near $T=0(\tilde{c}=0.01)$, for $\tilde{c}=0.90$, i.e., at the crossover temperature, as well as for $\tilde{c}=3.00$. They are computed for $\tilde{b}=0.11,0.44$, and 

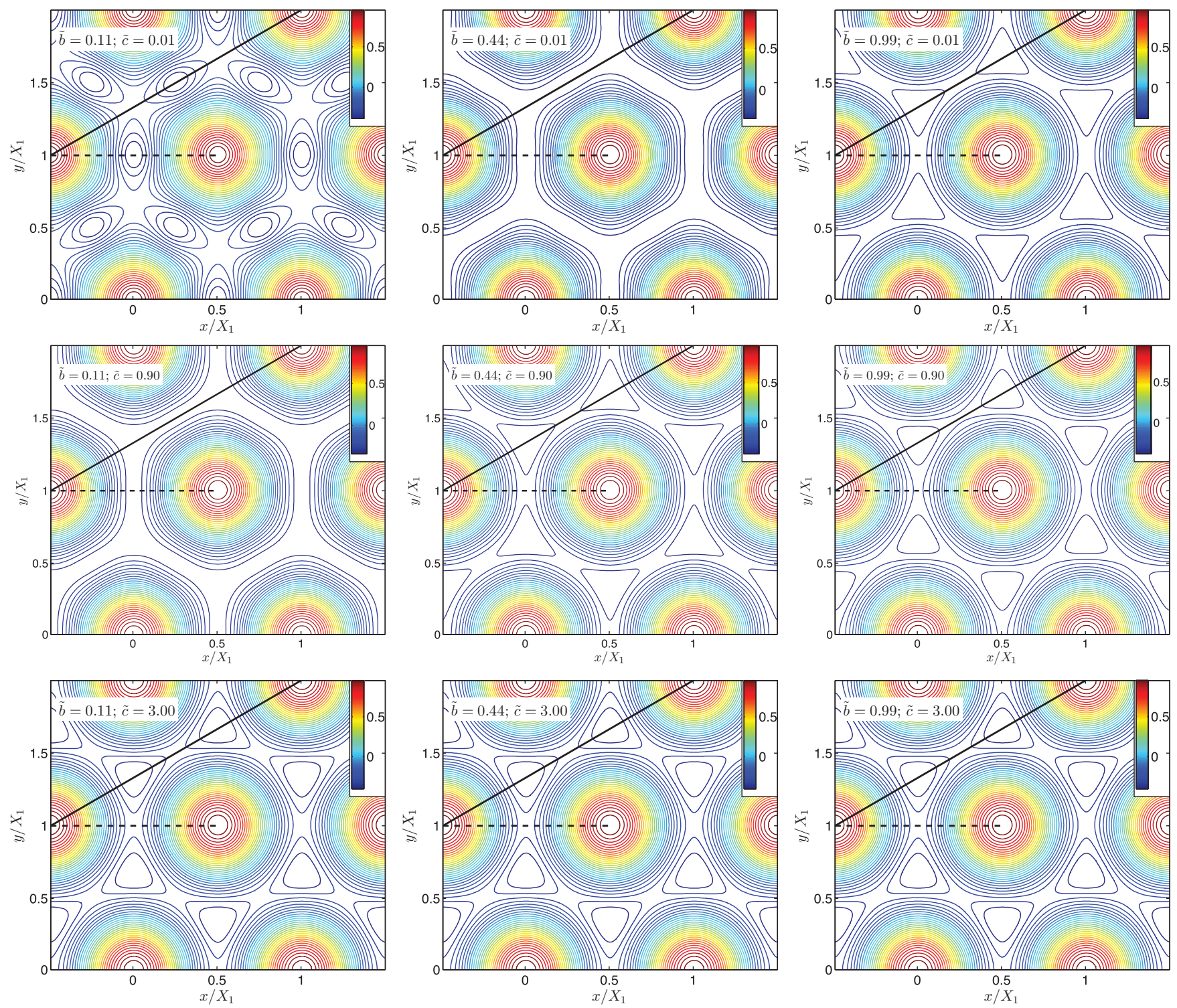

FIG. 4. (Color online) Contour plots of the field distribution for three values of $\tilde{c}(\tilde{c}=0.01,0.90$, and 3.00), and for $\tilde{b}$ significantly larger than zero: $\tilde{b}=0.11,0.44$, and 0.99 (corresponding to reduced fields $b=0.5,0.2$, and 0.1 , respectively).

0.99 , corresponding to the reduced fields $0.5,0.2$, and 0.1 , respectively. Focusing first on the maps at the top of the figure, i.e., for $\tilde{c}=0.01$, we note that the BCS regime, i.e., when Cooper pair diffraction matters, disappears when leaving the $B_{\mathrm{c} 2}$ phase boundary. This is clearly seen as the saddle point moves in between two vortex cores, as expected in the GL regime. Physically the distance between the cores becomes so large that the Cooper pair diffraction is no longer operative. The recovery of the GL features of the VL appears at a lower field if the temperature is increased, as seen from the maps at the crossover temperature. As noted from the maps at the bottom, i.e., at high temperature, their properties are field independent.

In Fig. 5 we show $D_{c}\left(B^{Z}\right)$, the field profiles along the dashed and solid lines in Fig. 4, and the form factors. While at $\tilde{c}=\tilde{c}_{\text {cross }}$ they are still weakly $\tilde{b}$ dependent, this is no more the case when $\tilde{c}=3.00$. This is obviously consistent with the field behavior of the maps, as seen in Fig. 4. At low temperature, i.e., at $\tilde{c}=0.01$, the shape of $D_{c}\left(B^{Z}\right)$, the field profiles, as well as the amplitude of the renormalized form factors, are strongly field dependent, confirming the results shown in Fig. 4. Only at high field is the behavior in the BCS regime observed.

\section{DISCUSSION AND CONCLUSIONS}

As pointed out by $\mathrm{U}$. Brandt et al. the approximate Gorkov equation used above is valid in the region of fields near $B_{\mathrm{c} 2}(T)$ where the magnetization vs field curve does not deviate appreciably from the straight line. ${ }^{10}$ For superconductors with a GL parameter $\kappa \sim 1$ this corresponds to a rather limited field range; however for $\kappa \gg 1$ it covers a substantial part of the VL phase. The validity of the model is related to two types of approximations which have been done for the derivations of the magnetization and form factor. We shall focus our discussion 

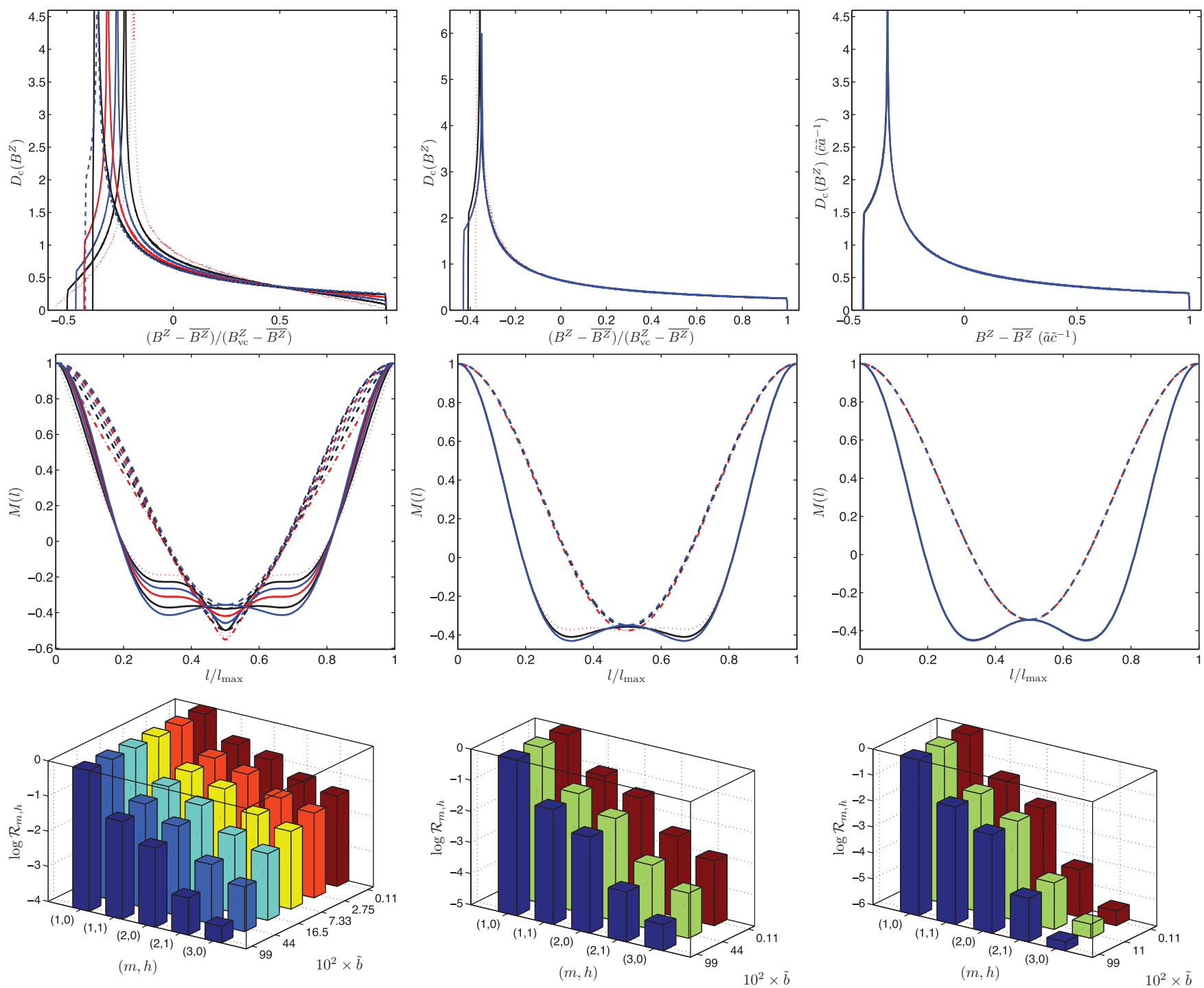

FIG. 5. (Color online) Field dependence of the component field distributions, field profiles, and form factors at low temperature- $\tilde{c}=0.01$ and $\tilde{b}=0.0011,0.0275,0.0733,0.165,0.44$, and 0.99 (corresponding to $b=0.99,0.8,0.6,0.4,0.2$, and 0.1 , respectively)-for the left column, at the crossover temperature- $\tilde{c}=0.90$ and $\tilde{b}=0.0011,0.44$, and $0.99(b=0.99,0.2$, and 0.1 , respectively $)$ in the middle column, and at high temperature $-\tilde{c}=3.00$ and $\tilde{b}=0.0011,0.11$, and $0.99(b=0.99,0.5$, and 0.1 , respectively $)$-in the right column.

on the latter quantity. The first type is inherent to the method and the second can be taken out if necessary.

We first recall the two approximations of the first type. The form factor is computed with an approximate Green's function. First, the effect of the field on the function is only described with a phase integral. This is the widely used semiclassical approximation. The ratio defined at Eq. (11) has to be smaller than 1 for this approximation to be valid. Second, the spatial variation of the induction is neglected. Therefore this cannot be valid if $B_{\text {ext }}$ is too close to $B_{\mathrm{c} 1}$.

We now discuss the second type of approximation. First, a spherical Fermi surface has been chosen. It should not be a problem to describe a superconductor with an anisotropic Fermi surface. However, it is probably possible only numerically. Second, up to now the conduction electron mean-free path $\ell_{\mathrm{mfp}}$ has been assumed to be infinite. Here we describe a method to account for the finite $\ell_{\mathrm{mfp}}$ value.
In the case of an isotropic impurity diffusion, the effect of these impurities can approximately be taken into account in the following way. First the Matsubara angular precession frequency $\omega_{\ell}$ in Eq. (10) is substituted by $\omega_{\ell}+1 /\left(2 \tau_{\text {imp }}\right)$ where $\tau_{\text {imp }}=\ell_{\mathrm{mfp}} / v_{\mathrm{F}}$. We identify $\tau_{\text {imp }}$ introduced here with $\tau_{\text {life }}$ used in Sec. III. Hence in the formula for the form factor $\omega_{\ell}$ has the meaning 28

$$
\omega_{\ell}=(2 \ell+1) \pi k_{\mathrm{B}} T / \hbar+1 /\left(2 \tau_{\mathrm{imp}}\right) .
$$

Second, $\Delta_{0}$ has to be renormalized. ${ }^{12}$ It is substituted by

$$
\frac{\Delta_{0}}{1-\varepsilon\left(\omega_{\ell}\right)}
$$

with

$$
\varepsilon\left(\omega_{\ell}\right)=\frac{1}{2} \frac{\Lambda}{\tau_{\mathrm{imp}} v_{\mathrm{F}}} \int_{0}^{\pi / 2} i v\left(\frac{i \omega_{\ell} \Lambda}{v_{\mathrm{F}} \sin \theta}\right) d \theta .
$$



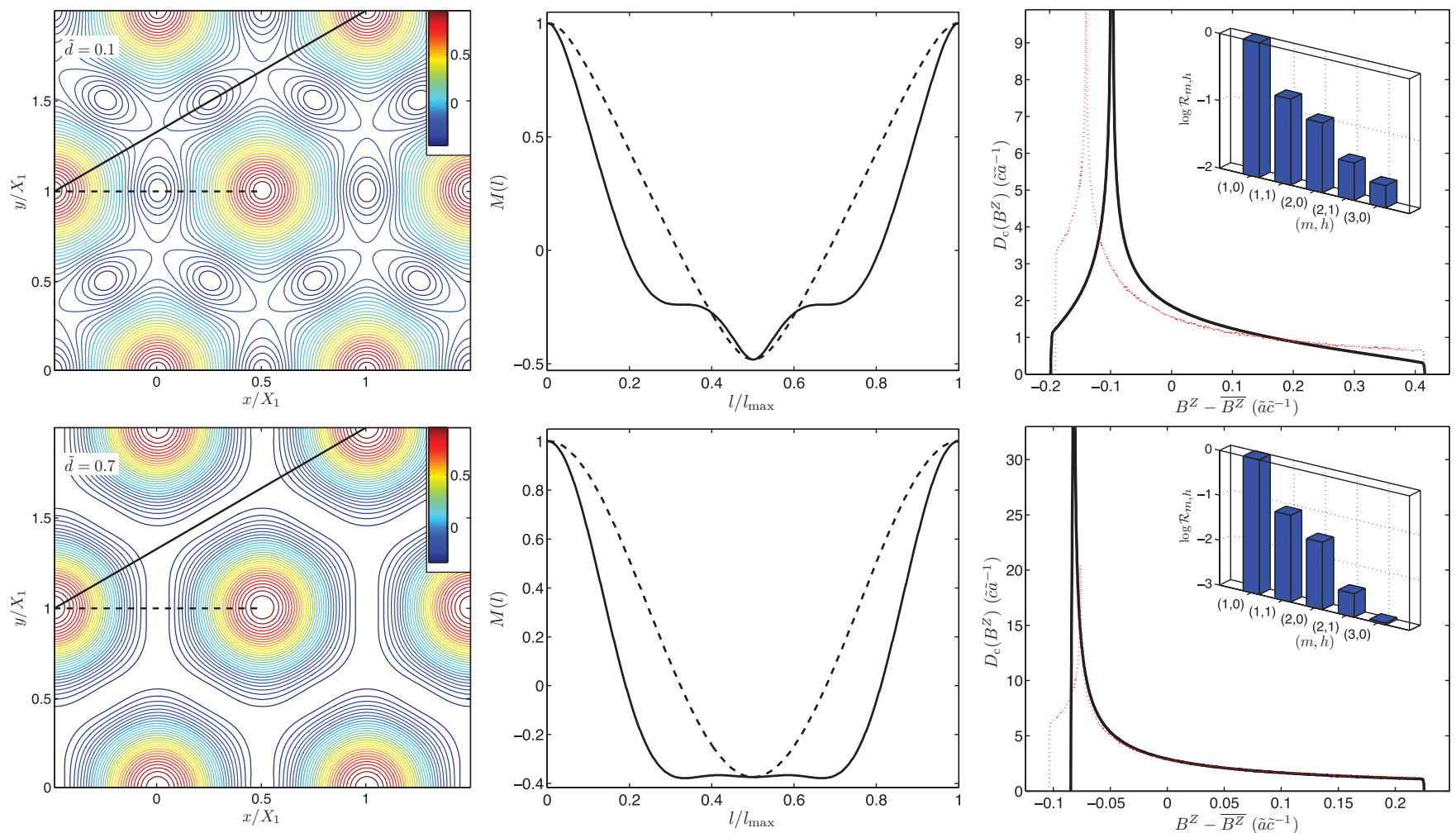

FIG. 6. (Color online) The same as in Fig. 2, but for $\tilde{d}=0.1$ and 0.7 and fixed $\tilde{b}=0.0011$ and $\tilde{c}=0.001$. The different plots show clearly that when the conditions for observation of a BCS type of $\mathrm{VL}$ are achieved (i.e., for $\tilde{b} \simeq 0$ and $\tilde{c} \simeq 0$ ) the transition from the BCS to the GL vortex lattice occurs at $\tilde{d} \simeq 0.7$. For $\tilde{d} \rightarrow 0$ the VL is in clean limit (see also Fig. 2) while for $\tilde{d} \rightarrow \infty$ it is of the GL type as in the high-temperature range; i.e., $\tilde{c} \rightarrow \infty$ (see Fig. 3 and Appendix E2).

Note, $\lim _{\tau_{\text {imp }} \rightarrow 0} \varepsilon\left(\omega_{\ell}\right)=1$. Since $\varepsilon\left(\omega_{\ell}\right)>0$, when the scattering is not too strong, i.e., when $\tau_{\text {imp }}$ is sufficiently long, the renormalization increases $\Delta_{0}$. This means that the PippardBCS coherence length decreases, as expected. Consistent with the region of validity of the form factor expression given by Eqs. (25) and (26), the proposed renormalization is only valid if $B_{\text {ext }}$ is not too far from $B_{\mathrm{c} 2}$. In the $B_{\mathbf{K}_{m, h}}^{Z}$ expression the renormalization occurs three times: twice explicitly and once through the variable $u_{\ell}$. As has been done in the clean limit, it is possible to write the $B_{\mathbf{K}_{m, h}}^{Z}$ expression in terms of a reduced number of parameters; see Appendix E 2 .

Eilenberger has derived approximate equations for the Green's functions for which a numerical method has been developed to solve them; see Refs. 8 and 29 and references therein. With this formalism the form factor can be computed with the effect of a finite $\ell_{\mathrm{mfp}}$ accounted for. ${ }^{30}$ It would be worthwhile to reproduce the analytical results described here with the numerical method and extend this study outside the region of validity of the analytical solution, i.e., far below $B_{\mathrm{c} 2}(T)$.

The linear $D_{\mathrm{c}}\left(B^{Z}\right)$ tail at low and high field and the relative large amplitude ratio $\mathcal{R}_{m, h}=\left|B_{\mathbf{K}_{m, h}}^{Z} / B_{\mathbf{K}_{1,0}}^{Z}\right|$ for Bragg's spots far from the center of the reciprocal space are consequences of the diffraction of the Cooper pairs on the vortex cores. Hence, a priori they should also be observed even for nonBCS-wave superconductors. For these exotic properties to be found, measurements have to be performed on very clean single-crystal superconductors (which is usually the case for high-temperature superconductors) at low temperature and for $B_{\text {ext }}$ sufficiently close to $B_{\mathrm{c} 2}$.

For the diffraction of carriers on a periodic VL to matter at low temperature, $\ell_{\mathrm{mfp}}$ should be substantially larger than the intervortex distance; i.e., $\tilde{d}=\Lambda / \ell_{\mathrm{mfp}} \ll 1$. For $\tilde{d} \gg 1$ no diffraction takes place and the VL has the GL profile similar to that shown in Fig. 3 (see Appendix E2) while for $\tilde{d}=0$ the superconductor is in the clean limit which was discussed above. In Fig. 6 we show contour plots of the spatial field distributions, field profiles, component field distributions, and form factors for intermediate values of $\tilde{d}=0.1$ and 0.7 with fixed $\tilde{c}=0.001$ and $\tilde{b}=0.0011$ (since $\tilde{b} \simeq 0$ and $\tilde{c} \simeq 0$ are optimal for the observation of the diffraction effects). For $\tilde{d}<$ 0.1 the characteristics of the VL are similar to that of clean VL. With increasing $\tilde{d}$ the exotic behavior of VL gradually vanishes and at $\tilde{d} \simeq 0.7$ the crossover takes place. This result differs only slightly from that obtained by E. H. Brandt using a nonlocal theory. ${ }^{6}$ This crossover depends on the combination of $\tilde{b}, \tilde{c}$, and $\tilde{d}$. In Fig. 7 plots for the crossover condition are given as a function of $\tilde{b}, \tilde{c}$, and $\tilde{d}$ ( $\tilde{b}$ vs $\tilde{c}$ for different $\tilde{d}$ values). This conclusive figure illustrates the following natural condition for the observation of the exotic VL behavior due to diffraction: The three length scales $\xi^{T}, \xi^{B}$, and $\ell_{\mathrm{mfp}}$ should be significantly larger than the intervortex distance [here, $\xi^{T}=$ $\hbar v_{\mathrm{F}} /\left(2 \pi k_{\mathrm{B}} T\right), \xi^{B}=\hbar v_{\mathrm{F}} / \pi \Delta_{0}$; see Appendix E 1$]$.

In conclusion, we have reviewed the previous works of Delrieu on the exotic behavior of the vortex lattice (VL) at high field and low temperature. It is the consequence of the Cooper 


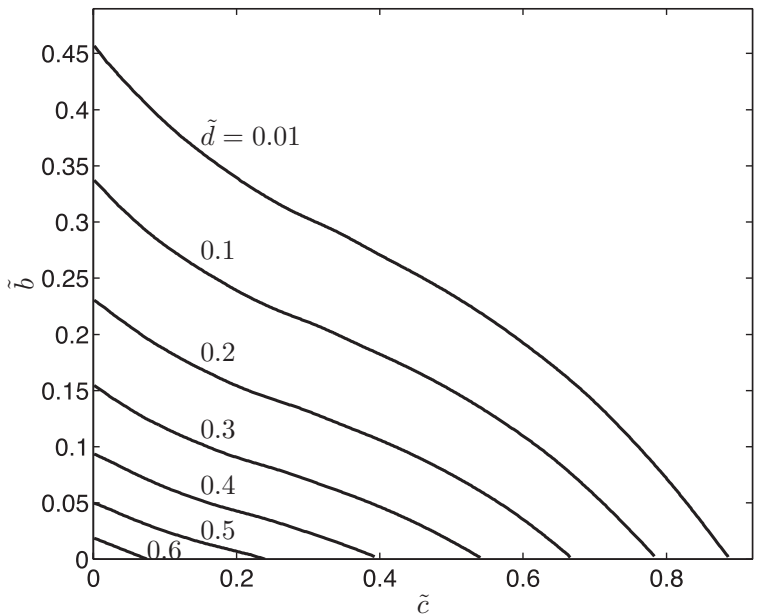

FIG. 7. Plots of $\tilde{b}$ vs $\tilde{c}$ for $\tilde{d}=0.01,0.1,0.2,0.3,0.4,0.5$, and 0.6 where the condition of equal fields at saddle and minimal points is satisfied (the crossover condition; see, e.g., top panel of Fig. 3).

pair diffraction on the periodic VL potential. Analytical and numerical results for the magnetization and form factors are derived using the Green's function formalism. In agreement with previous works of $\mathrm{E}$. H. Brandt where a nonlocal theory of superconductivity was utilized (see, e.g., Refs. 6 and 26 and references therein), we find a set of conditions for the observation of this exotic behavior of the VL. Namely, the intervortex distance should be significantly smaller than each three length parameters: $\xi^{T}, \xi^{B}$, and $\ell_{\mathrm{mfp}}$ (see the text). An expression for the standard deviation of the component field distribution has been derived. The results of a numerical study of the form factors $\left(B_{\mathbf{K}_{m, h}}^{Z}\right)$, field map, and field distribution $\left[D_{\mathrm{c}}\left(B^{Z}\right)\right]$ have been presented for a broad range of applied field $B_{\text {ext }}$ and covering the whole range of temperatures from $T=0$ up to $T_{\mathrm{c} 0}$. In addition, the effect of impurities was studied. This has enabled us to determine features which distinguish GL from low-temperature BCS vortex lattices. The behaviors of the experimentally accessible $D_{\mathrm{c}}\left(B^{Z}\right)$ and $\left|B_{\mathbf{K}_{m, h}}^{Z} / B_{\mathbf{K}_{1,0}}^{Z}\right|$ quantities versus the normalized temperature and external field have been exposed. These results should at least apply to niobium for $\mathbf{B}_{\text {ext }} \|$ [111], and maybe other classical BCS superconductors such as vanadium. This analysis will help in searching the exotic VL behavior using the SANS, $\mu \mathrm{SR}$, and NMR techniques, since the Cooper pair diffraction is not restricted to the BCS theory and the conditions of the diffraction presented above can well be satisfied by most of the high-temperature superconductors.

\section{ACKNOWLEDGMENTS}

We are grateful to P. Dalmas de Réotier for helpful discussions and a careful reviewing of this manuscript and M. Houzet for a useful discussion on the validity of the GL model.

\section{APPENDIX A: THE $v(z)$ FUNCTION AND THE RELATED DAWSON INTEGRAL}

The magnetization and the form factor for the field near $B_{\mathrm{c} 2}$ are expressed in terms of the function $v(z)$ which is related to the so-called complementary error function $\operatorname{erfc}(z):^{31}$

$$
v(z)=\frac{1}{\sqrt{\pi}} \int_{-\infty}^{\infty} \frac{\exp \left(-t^{2}\right)}{z-t} d t=\frac{\sqrt{\pi}}{i} \exp \left(-z^{2}\right) \operatorname{erfc}(-i z) .
$$

We have the relation

$$
v(-z)=-2 \sqrt{\pi} i \exp \left(-z^{2}\right)-v(z) .
$$

Here $z$ and $t$ are complex and real variables, respectively. In our case $z=i x$, where $x$ is real, and in the asymptotic large- $x$ limit

$$
i v(i x)=\frac{1}{x}\left[1-\frac{1}{2 x^{2}}+\mathcal{O}\left(\frac{1}{x^{4}}\right)\right] .
$$

In general, we have the relation

$$
v^{\prime}(z)=-2 z v(z)+2,
$$

where $v^{\prime}(z)=d v(z) / d z$. Combining the two previous equations, we derive

$$
v^{\prime}(i x)=1 / x^{2}, \quad x \rightarrow \infty
$$

and

$$
i v^{\prime \prime}(i x)=-2 / x^{3}, \quad x \rightarrow \infty .
$$

The $i v(i x)$ function is bounded as follows:

$$
-\left(x-\sqrt{x^{2}+2}\right)<i v(i x)<-\frac{\pi}{2}\left(x-\sqrt{x^{2}+4 / \pi}\right) .
$$

We note the asymptotic limit of the Dawson integral:

$$
\exp \left(-x^{2}\right) \int_{0}^{x} \exp \left(t^{2}\right) d t=\frac{1}{2 x}, \quad x \rightarrow \infty .
$$

\section{APPENDIX B: EVALUATION OF $a(\partial / \partial a)\left(u_{\ell} / a\right)$}

Here we evaluate

$$
A=a \frac{\partial}{\partial a}\left(\frac{u_{\ell}}{a}\right),
$$

which is required to derive the magnetization from the free energy. It is easily found that

$$
A=\frac{\partial u_{\ell}}{\partial a}-\frac{u_{\ell}}{a} .
$$

To compute $\partial u_{\ell} / \partial a$, we first note that according to Eq. (16) we can write

$$
-u_{\ell}+2 \hbar \omega_{\ell} a+\Delta_{0}^{2} a^{2} i v\left(i u_{\ell}\right)=f\left(u_{\ell}, a\right)=0 .
$$

This implies that

$$
\frac{\partial u_{\ell}}{\partial a}=-\frac{\partial f / \partial a}{\partial f / \partial u_{\ell}}=2 \frac{\hbar \omega_{\ell}+\Delta_{0}^{2} a i v\left(i u_{\ell}\right)}{1+\Delta_{0}^{2} a^{2} v^{\prime}\left(i u_{\ell}\right)},
$$

where $v^{\prime}(z)=d v(z) / d z$. This means that

$$
A=\Delta_{0}^{2} a \frac{-u_{\ell} v^{\prime}\left(i u_{\ell}\right)+i v\left(i u_{\ell}\right)}{1+\Delta_{0}^{2} a^{2} v^{\prime}\left(i u_{\ell}\right)} .
$$

Now we note the relation

$$
\frac{\partial\left[u_{\ell} i v\left(i u_{\ell}\right)\right]}{\partial\left(\hbar \omega_{\ell}\right)}=\frac{\partial u_{\ell}}{\partial\left(\hbar \omega_{\ell}\right)}\left[i v\left(i u_{\ell}\right)-u_{\ell} v^{\prime}\left(i u_{\ell}\right)\right] .
$$


Since

$$
\frac{\partial u_{\ell}}{\partial\left(\hbar \omega_{\ell}\right)}=-\frac{\partial f / \partial\left(\hbar \omega_{\ell}\right)}{\partial f / \partial u_{\ell}}=\frac{2 a}{1+\Delta_{0}^{2} a^{2} v^{\prime}\left(i u_{\ell}\right)},
$$

we derive

$$
\frac{\partial\left[u_{\ell} i v\left(i u_{\ell}\right)\right]}{\partial\left(\hbar \omega_{\ell}\right)}=\frac{2 a\left[i v\left(i u_{\ell}\right)-u_{\ell} v^{\prime}\left(i u_{\ell}\right)\right]}{1+\Delta_{0}^{2} a^{2} v^{\prime}\left(i u_{\ell}\right)} .
$$

Combining the previous equation with Eq. (B5), we obtain

$$
A=\frac{\Delta_{0}^{2}}{2} \frac{\partial\left[u_{\ell} i v\left(i u_{\ell}\right)\right]}{\partial\left(\hbar \omega_{\ell}\right)} .
$$

Recalling the relation given at Eq. (A4), we finally derive

$$
A=-\frac{\Delta_{0}^{2}}{4} i v^{\prime \prime}\left(i u_{\ell}\right) \frac{\partial u_{\ell}}{\partial\left(\hbar \omega_{\ell}\right)} \text {. }
$$

\section{APPENDIX C: LOW-TEMPERATURE ASYMPTOTIC LIMIT OF THE MAGNETIZATION}

We start from Eq. (21). When the temperature is very small, according to Eq. (10) it is justified to replace the sum $2 \pi k_{\mathrm{B}} T \sum_{\ell}$ by the integral $\int_{u_{0}}^{\infty} d(\hbar \omega)$. Since, according to Eq. (A5), $v^{\prime}\left(i u_{\ell}\right)$ vanishes when $\ell \rightarrow \infty$,

$$
M=-\frac{N_{0} \Delta_{0}^{2}}{4 \overline{B^{Z}}} \int_{0}^{\pi / 2} \sin (\theta) v^{\prime}\left(i u_{0}\right) d \theta .
$$

As a Matsubara frequency vanishes with the temperature,

$$
u_{0}=\frac{\Delta_{0}^{2} \Lambda^{2}}{\hbar^{2} v_{\mathrm{F}}^{2} \sin ^{2} \theta} i v\left(i u_{0}\right) \text {. }
$$

Because we are focusing on the field region near $B_{\mathrm{c} 2}$, except for a small domain for which $\theta$ can be small, we can take $u_{0}=0$. Using Eq. (A4), we then get $v^{\prime}(0)=2$, and finally derive the result written in Eq. (22).

\section{APPENDIX D: ASYMPTOTIC LIMITS OF THE FORM FACTOR}

Here we determine analytically two asymptotic limits of the form factor $B_{\mathbf{K}_{m, h}}^{Z}$ starting from Eqs. (25) and (26). We shall first study the high-temperature limit.

\section{The behavior near $T_{\mathrm{c} 0}$}

When approaching $T_{\mathrm{c} 0}$ from below, $\overline{B^{Z}}$ vanishes as does $B_{\mathrm{c} 2}$. Hence $\Lambda$ is getting very large. Referring to Eq. (27), this means that $u_{\ell}$ is large. This has two consequences. First, it is justified to consider the $u_{\ell} \gg n_{\mathbf{K}_{m, h}}$ limit for the numerator of the fraction in $g_{\ell}(\theta)$. Recalling the Taylor expansion of a function,

$$
\begin{aligned}
& i v\left(i u_{\ell}+i n_{\mathbf{K}_{m, h}}\right)+i v\left(i u_{\ell}-i n_{\mathbf{K}_{m, h}}\right)-2 i v\left(i u_{\ell}\right) \\
& \quad \approx-n_{\mathbf{K}_{m, h}}^{2} i v^{\prime \prime}\left(i u_{\ell}\right) .
\end{aligned}
$$

Second, let us now focus on the denominator, in particular on the second term. Because $u_{\ell}$ is large, for $i v\left(i u_{\ell}\right)$ we can use the first term of its expansion given by Eq. (A3). From Eq. (27) we then get

$$
u_{\ell}=2 \omega_{\ell} \frac{\Lambda}{v_{\mathrm{F}} \sin \theta}+\frac{\Delta_{0}^{2} \Lambda^{2}}{\hbar^{2} v_{\mathrm{F}}^{2} \sin ^{2} \theta} \frac{1}{u_{\ell}} .
$$

Therefore, to a good approximation near $T_{\mathrm{c} 0}$,

$$
u_{\ell}=2 \omega_{\ell} \frac{\Lambda}{v_{\mathrm{F}} \sin \theta} .
$$

Using Eq. (A5), we deduce

$$
\frac{\Delta_{0}^{2} \Lambda^{2}}{\hbar^{2} v_{\mathrm{F}}^{2} \sin ^{2} \theta} v^{\prime}\left(i u_{\ell}\right)=\left(\frac{\Delta_{0}}{2 \hbar \omega_{\ell}}\right)^{2}=\left[\frac{\Delta_{0}}{(2 \ell+1) 2 \pi k_{\mathrm{B}} T}\right]^{2} .
$$

Hence, since $\Delta_{0}$ vanishes on approaching $T_{\mathrm{c} 0}$, the second term of the denominator in Eq. (26) becomes negligible relative to 1 .

Using the two previous results, we derive from Eqs. (25) and (26) the asymptotic behavior of $B_{\mathbf{K}_{m, h}}^{Z}$ near $T_{\mathrm{c} 0}$ :

$$
\begin{aligned}
B_{\mathbf{K}_{m, h}}^{Z}= & \frac{2 \pi^{2} \mu_{0} N_{0} \Delta_{0}^{2} k_{\mathrm{B}} T}{\overline{B^{Z}}}(-1)^{m h} \exp \left(-n_{\mathbf{K}_{m, h}}^{2}\right) \\
& \times \sum_{\ell=0}^{\infty} \int_{0}^{\pi / 2} \sin (\theta) i v^{\prime \prime}\left(i u_{\ell}\right) \frac{\partial u_{\ell}}{\partial\left(\hbar \omega_{\ell}\right)} d \theta .
\end{aligned}
$$

Recalling Eq. (21) we find, as expected, that $B_{\mathbf{K}_{m, h}}^{Z}$ is proportional to the magnetization as written explicitly at Eq. (28).

\section{Low-temperature limit}

Since we are interested in this work in the limit for which the field is near $B_{\mathrm{c} 2}, \Delta_{0}$ is small. According to Appendix A, $v^{\prime}\left(i u_{\ell}\right)$ is bounded. Therefore, except for small $\theta$ values, we can neglect the second term in the denominator of the $g_{\ell}(\theta)$ function relative to one. As done for the study of the lowtemperature limit of the magnetization, we can substitute the $\operatorname{sum} 2 \pi k_{\mathrm{B}} T \sum_{\ell}$ with the integral $\int_{u_{0}}^{\infty} d(\hbar \omega)$. This gives

$$
B_{\mathbf{K}_{m, h}}^{Z}=-\frac{\pi N_{0} \mu_{0} \Delta_{0}^{2}}{\overline{B^{Z}}} \frac{(-1)^{m h}}{n_{\mathbf{K}_{m, h}}^{2}} \int_{0}^{\pi / 2} \sin (\theta) h_{\ell}(\theta) d \theta,
$$

with

$$
\begin{aligned}
h_{\ell}(\theta)= & \exp \left(-n_{\mathbf{K}_{m, h}}^{2}\right)\left[\int_{u_{0}}^{\infty} i v\left(i x+i n_{\mathbf{K}_{m, h}}\right) d x\right. \\
& \left.+\int_{u_{0}}^{\infty} i v\left(i x-i n_{\mathbf{K}_{m, h}}\right) d x-2 \int_{u_{0}}^{\infty} i v(i x) d x\right] .
\end{aligned}
$$

In the first and second terms we use the new variables $t=x+n_{\mathbf{K}_{m, h}}$ and $t=x-n_{\mathbf{K}_{m, h}}$, respectively, and split the integration $\int_{u_{a}}^{\infty} i v(i t) d t$ into $\int_{u_{a}}^{u_{0}} i v(i t) d t+\int_{u_{0}}^{\infty} i v(i t) d t$, where $u_{a}=u_{0}+n_{\mathbf{K}_{m, h}}$ and $u_{a}=u_{0}-n_{\mathbf{K}_{m, h}}$ for the first and the second terms, respectively. The two integrals $\int_{u_{0}}^{\infty} i v(i t) d t$ cancel the third term. As a result we obtain the following relation:

$$
\begin{aligned}
h_{\ell}(\theta)= & \exp \left(-n_{\mathbf{K}_{m, h}}^{2}\right)\left[\int_{u_{0}+n_{\mathbf{K}_{m, h}}}^{u_{0}} i v(i t) d t\right. \\
& \left.+\int_{u_{0}-n_{\mathbf{K}_{m, h}}}^{u_{0}} i v(i t) d t\right] .
\end{aligned}
$$

Let us study the function $h_{\ell}(\theta)$. Since the field is near $B_{\mathrm{c} 2}$ and we are at low temperature, $u_{0} \simeq 0$. Then setting $u_{0}=0$ 
and using Eq. (A2), we get

$$
\begin{aligned}
h_{\ell}(\theta)= & \exp \left(-n_{\mathbf{K}_{m, h}}^{2}\right)\left[2 \sqrt{\pi} \int_{0}^{n_{\mathbf{K}_{m, h}}} \exp \left(t^{2}\right) d t\right. \\
& \left.-2 \int_{0}^{n_{\mathbf{K}_{m, h}}} i v(i t) d t\right] .
\end{aligned}
$$

From Eq. (A7),

$$
\int_{0}^{n_{\mathbf{K}_{m, h}}} i v(i t) d t<-\frac{\pi}{2} \int_{0}^{n_{\mathbf{K}_{m, h}}^{2}}\left(t-\sqrt{t^{2}+4 / \pi}\right) d t .
$$

Hence the second term of $h_{\ell}(\theta)$, i.e., $2 \exp \left(-n_{\mathbf{K}_{m, h}}^{2}\right) \int_{0}^{n_{\mathbf{K}_{m, h}}} i v(i t) d t$, is negligible if $n_{\mathbf{K}_{m, h}}^{2}$ is sufficiently large. Since the first term is proportional to the Dawson integral, using Eq. (A8) we finally derive the $h_{\ell}(\theta)$ asymptotic limit:

$$
h_{\ell}(\theta)=\sqrt{\pi} / n_{\mathbf{K}_{m, h}} \quad \text { when } \quad n_{\mathbf{K}_{m, h}} \rightarrow \infty .
$$

Combining this result with Eq. (D6), we derive the asymptotic limit written at Eq. (31).

\section{APPENDIX E: THE FORM FACTOR IN TERMS OF A REDUCED NUMBER OF PARAMETERS}

The original $B_{\mathbf{K}_{m, h}}^{Z}$ expression given by Eqs. (25)-(27) depends on three material parameters $\Delta_{0}, N_{0}, v_{F}$, and two experimental parameters $T$ and $B_{\text {ext }}$ since $\overline{B^{Z}} \simeq B_{\text {ext }}$. In the next subsection we show that in fact it is a function of only three independent parameters. Even more interesting, only one of these parameters has to be varied to study the region close to the $B_{\mathrm{c} 2}(T)$ boundary. The second subsection gives a formula for $B_{\mathbf{K}_{m, h}}^{Z}$ when the electronic mean-free path is a finite. The dirty limit is studied.

\section{The form factor in the clean limit}

It is easily shown that the formula for $B_{\mathbf{K}_{m, h}}^{Z}$ can be written as follows:

$$
B_{\mathbf{K}_{m, h}}^{Z}=\tilde{a} \frac{(-1)^{m h} \exp \left(-n_{\mathbf{K}_{m, h}}^{2}\right)}{n_{\mathbf{K}_{m, h}}^{2}} \sum_{\ell=0}^{\infty} \int_{0}^{\pi / 2} f_{\ell}(\theta) d \theta,
$$

where

$$
f_{\ell}(\theta)=\frac{i v\left(i u_{\ell}+i n_{\mathbf{K}_{m, h}}\right)+i v\left(i u_{\ell}-i n_{\mathbf{K}_{m, h}}\right)-2 i v\left(i u_{\ell}\right)}{\left[1+\tilde{b} \frac{v^{\prime}\left(i u_{\ell}\right)}{\sin ^{2} \theta}\right]^{3}} .
$$

We have used the analytical expression of $\partial u_{\ell} / \partial\left(\hbar \omega_{\ell}\right)$ written in Eq. (B7). The $u_{\ell}$ 's are found to be the solution of the equation

$$
u_{\ell}=\tilde{c} \frac{(2 \ell+1)}{\sin \theta}+\tilde{b} \frac{i v\left(i u_{\ell}\right)}{\sin ^{2} \theta} .
$$

The proportionality coefficient $\tilde{a}$ is in magnetic induction units. It is written as follows:

$$
\tilde{a}=-\mu_{0} \pi N_{0} \Delta_{0}^{2} 2 \pi k_{\mathrm{B}} T \frac{2 \Lambda}{\overline{B^{Z}} \hbar v_{\mathrm{F}}}=-\mu_{0} \pi N_{0} \Delta_{0}^{2} \frac{2 \tilde{c}}{\overline{B^{Z}}} .
$$

We have also introduced the dimensionless parameters

$$
\tilde{b}=\left(\Lambda / \pi \xi^{B}\right)^{2}
$$

and

$$
\tilde{c}=\Lambda / \xi^{T}
$$

where we have defined the temperature and field dependent length scale parameters $\xi^{T}=\hbar v_{\mathrm{F}} /\left(2 \pi k_{\mathrm{B}} T\right)$ and $\xi^{B}=$ $\hbar v_{\mathrm{F}} / \pi \Delta_{0}$, respectively. Therefore $B_{\mathbf{K}_{m, h}}^{Z}$ has been written in terms of three parameters: $\tilde{a}, \tilde{b}$, and $\tilde{c}$. Interestingly, $\tilde{b}$ is vanishingly small when $\Delta_{0} \rightarrow 0$. Then the second term of the denominator of Eq. (E2) is negligible and $\tilde{a}$ becomes small. As a consequence, as expected, the form factor is also small.

There is interest in studying the temperature dependence of $\tilde{b}$ and $\tilde{c}$ near the phase boundary $B_{\mathrm{c} 2}(T)$, in particular their asymptotes. We first note that $\Delta_{0} \rightarrow 0$. In addition, by the definition of the second critical field given by Eq. (20), $\Lambda \simeq \xi_{\mathrm{GL}}$ since $\overline{B^{Z}} \simeq B_{\mathrm{c} 2}$. Let us investigate the $T \rightarrow 0$ limit. According to Eq. (19) we derive $\Lambda \simeq \xi_{0} / 0.96$. Hence $\Lambda$ is finite, and therefore $\tilde{b} \rightarrow 0$. Since $\xi^{T}$ diverges, we also derive $\tilde{c} \rightarrow 0$. Concerning the $T \rightarrow T_{\mathrm{c} 0}$ limit, we note that $\Lambda$ diverges as does $\xi_{\mathrm{GL}}$. This first means that $\tilde{b}$ is the ratio of two large numbers. Numerically we find $\tilde{b} \ll 1$. An example is given in Sec. VII. Second, as $\xi^{T}$ is finite, $\tilde{c} \rightarrow \infty$.

According to this discussion, in the limit $T \rightarrow T_{\mathrm{c} 0}$ and near $B_{\mathrm{c} 2}$, we can set $\tilde{c} \rightarrow \infty$ and $\tilde{b} \rightarrow 0$. This means that

$$
u_{\ell}=\tilde{c} \frac{(2 \ell+1)}{\sin \theta},
$$

and using Eqs. (D1) and (A6),

$$
f_{\ell}(\theta) \simeq-n_{\mathbf{K}_{m, h}}^{2} i v^{\prime \prime}\left(i u_{\ell}\right) \simeq 2 n_{\mathbf{K}_{m, h}}^{2} \frac{\sin ^{3} \theta}{\tilde{c}^{3}(2 \ell+1)^{3}} .
$$

Taking these results into account, we derive

$$
\begin{aligned}
B_{\mathbf{K}_{m, h}}^{Z}= & 2 \frac{\tilde{a}}{\tilde{c}^{3}}(-1)^{m h} \exp \left(-n_{\mathbf{K}_{m, h}}^{2}\right)\left[\sum_{\ell=0}^{\infty}(2 \ell+1)^{-3}\right] \\
& \times\left[\int_{0}^{\pi / 2} \sin ^{3} \theta d \theta\right] \\
= & 1.4024 \frac{\tilde{a}}{\tilde{c}^{3}}(-1)^{m h} \exp \left(-n_{\mathbf{K}_{m, h}}^{2}\right) .
\end{aligned}
$$

Here, we have used the results $\int_{0}^{\pi / 2} \sin ^{3} \theta d \theta=2 / 3$ and $\sum_{0}^{\infty}(2 \ell+1)^{-3}=7 \zeta(3) / 8=1.0518$, with $\zeta(s)$ denoting the Riemann zeta function. As can be seen from the last equation, for $T \rightarrow T_{\mathrm{c} 0}$ the form factor converges to the GL solution. It is proportional to the factor $\tilde{a} / \tilde{c}^{3}$. On the other hand, as can be seen from Eq. (31), in the low-temperature limit near $B_{\text {ext }} \simeq B_{\text {c2 }}$ (i.e., $\tilde{c} \rightarrow 0$ and $\tilde{b} \rightarrow 0$ ) $B_{\mathbf{K}_{m, h}}^{Z}$ is proportional to $\tilde{a} / \tilde{c}$ :

$$
B_{\mathbf{K}_{m, h}}^{Z}=\frac{\sqrt{\pi}}{2} \frac{\tilde{a}}{\tilde{c}} \frac{(-1)^{m h}}{n_{\mathbf{K}_{m, h}}^{3}} .
$$

Thus, the two limits being proportional to $\tilde{a} / \tilde{c}$, it is convenient to use this field scale as units of field.

In conclusion, considering the region very close to $B_{\mathrm{c} 2}(T)$ and the limits near $T_{\mathrm{c} 0}$ and $T=0$, we find that $\tilde{b}$ is very small. On the other hand, $\tilde{c}$ is large in the first limit and negligible in the second. Consequently $B_{\mathbf{K}_{m, h}}^{Z}$, and therefore the field map and distribution, is expected to strongly depend on $\tilde{c}$. This fact is used in Sec. VII for the study of the crossover from GL to BCS vortex structures. 


\section{The form factor for a finite mean-free path}

In the case of a finite electronic mean-free-path, i.e., of a finite scattering rate $1 / \tau_{\text {imp }}$, the form factor depends on the four parameters $\tilde{a}, \tilde{b}, \tilde{c}$, and $\tilde{d}$ as follows:

$$
B_{\mathbf{K}_{m, h}}^{Z}=\tilde{a} \frac{(-1)^{m h} \exp \left(-n_{\mathbf{K}_{m, h}}^{2}\right)}{n_{\mathbf{K}_{m, h}}^{2}} \sum_{\ell=0}^{\infty} \int_{0}^{\pi / 2} \frac{f_{\ell}(\theta)}{\left(1-\varepsilon_{\ell}\right)^{2}} d \theta,
$$

where

$$
f_{\ell}(\theta)=\frac{i v\left(i u_{\ell}+i n_{\mathbf{K}_{m, h}}\right)+i v\left(i u_{\ell}-i n_{\mathbf{K}_{m, h}}\right)-2 i v\left(i u_{\ell}\right)}{\left[1+\tilde{b} \frac{v^{\prime}\left(i u_{\ell}\right)}{\left(1-\varepsilon_{\ell}\right)^{2} \sin ^{2} \theta}\right]^{3}} .
$$

The $u_{\ell}$ 's are found to be the solution of the equation:

$$
u_{\ell}=\frac{\tilde{c}(2 \ell+1)+\tilde{d}}{\sin \theta}+\frac{\tilde{b}}{\left(1-\varepsilon_{\ell}\right)^{2} \sin ^{2} \theta} i v\left(i u_{\ell}\right),
$$

with

$$
\varepsilon_{\ell}=\frac{1}{2} \tilde{d} \int_{0}^{\pi / 2} i v\left(i \frac{\tilde{c}(2 \ell+1)+\tilde{d}}{2 \sin \theta}\right) d \theta .
$$

Note, $\lim _{\tilde{d} \rightarrow \infty} \varepsilon_{\ell}=1$. The parameters $\tilde{a}, \tilde{b}$, and $\tilde{c}$ are the same as in the clean limit case. We have defined the dimensionless scattering parameter,

$$
\tilde{d}=\frac{\Lambda}{v_{\mathrm{F}} \tau_{\text {imp }}} \simeq \frac{1}{b^{1 / 2}} \frac{\xi_{\mathrm{GL}}}{v_{\mathrm{F}} \tau_{\text {imp }}},
$$

which is approximately the ratio of intervortex distance to the electronic mean-free path. Hence, relative to the clean limit case, the effect of impurities and defects is taken into account with only a single new parameter, i.e., $\tilde{d}$.
In the dirty limit we have $\tilde{d} \gg 1$. This implies

$$
u_{\ell} \simeq \frac{\tilde{c}(2 \ell+1)+\tilde{d}}{\sin \theta} .
$$

Equation (E14) converges to

$$
\varepsilon_{\ell}=\frac{1}{2} \tilde{d} \int_{0}^{\pi / 2} \frac{2 \sin \theta}{\tilde{c}(2 \ell+1)+\tilde{d}} d \theta=\frac{\tilde{d}}{\tilde{c}(2 \ell+1)+\tilde{d}} .
$$

Here we used Eq. (A3) and $\int_{0}^{\pi / 2} \sin x d x=1$. Therefore,

$$
\left(1-\varepsilon_{\ell}\right)^{-2}=\left(\frac{2 \ell+1+\tilde{d} / \tilde{c}}{2 \ell+1}\right)^{2} .
$$

The denominator of Eq. (E12) converges to

$$
\left[1+\tilde{b} \frac{v^{\prime}\left(i u_{\ell}\right)}{\left(1-\varepsilon_{\ell}\right)^{2} \sin ^{2} \theta}\right]^{3} \simeq\left[1+\frac{\tilde{b}}{\tilde{c}^{2}} \frac{1}{(2 \ell+1)^{2}}\right]^{3},
$$

and using Eqs. (D1) and (A6),

$$
f_{\ell}(\theta) \simeq 2 n_{\mathbf{K}_{m, h}}^{2} \frac{\sin ^{3} \theta}{\left[(\tilde{c}(2 \ell+1)+\tilde{d})\left(1+\frac{\tilde{b}}{\tilde{c}^{2}} \frac{1}{(2 \ell+1)^{2}}\right)\right]^{3}} .
$$

This means for the form factor,

$$
B_{\mathbf{K}_{m, h}}^{Z} \simeq \tilde{A}(-1)^{m h} \exp \left(-n_{\mathbf{K}_{m, h}}^{2}\right),
$$

with

$$
\begin{aligned}
\tilde{A}= & \frac{4}{3} \tilde{a} \tilde{c}^{3} \sum_{\ell=0}^{\infty}\left[(2 \ell+1+\tilde{d} / \tilde{c})^{-1}(2 \ell+1)^{-2}\right. \\
& \left.\times\left[\tilde{c}^{2}+\tilde{b} /(2 \ell+1)^{2}\right]^{-3}\right] .
\end{aligned}
$$

Thus, as expected, no matter the temperature, if the scattering parameter $\tilde{d}$ is large the VL properties are described by the GL model.
${ }^{1}$ M. Tinkham, Introduction to Superconductivity (McGraw-Hill, New York, 1996).

${ }^{2}$ I. Landau and H. Keller, Physica C 466, 131 (2007).

${ }^{3}$ J. E. Sonier, Rep. Prog. Phys. 70, 1717 (2007).

${ }^{4}$ J. M. Delrieu, J. Low Temp. Phys. 6, 197 (1972).

${ }^{5}$ E. H. Brandt, Phys. Status Solidi B 64, 467 (1974).

${ }^{6}$ E. H. Brandt, Phys. Status Solidi B 65, 469 (1974).

${ }^{7}$ E. H. Brandt, Phys. Status Solidi B 77, 105 (1976).

${ }^{8}$ U. Klein, J. Low Temp. Phys. 69, 1 (1987).

${ }^{9}$ L. P. Gor'kov, Zh. Eksp. Teor. Fiz. 36, 1918 (1959) [Sov. Phys. JETP 9, 1364 (1959)].

${ }^{10}$ U. Brandt, W. Pesch, and L. Tewordt, Z. Phys. 201, 209 (1967).

${ }^{11}$ U. Brandt, Phys. Lett. A 29, 568 (1969).

${ }^{12}$ J. M. Delrieu, Ph.D. thesis, University of Paris-Orsay, 1974.

${ }^{13}$ A. Kung, Phys. Rev. Lett. 25, 1006 (1970).

${ }^{14}$ D. Herlach, G. Majer, J. Major, J. Rosenkranz, M. Schmolz, W. Schwarz, A. Seeger, W. Templ, E. Brandt, U. Essmann et al., Hyperfine Interact. 63, 41 (1990).

${ }^{15}$ A. Yaouanc, P. Dalmas de Réotier, and E. H. Brandt, Phys. Rev. B 55, 11107 (1997).

${ }^{16}$ N. R. Werthamer, in Superconductivity, edited by R. D. Parks, Vol. 1 (Marcel Dekker, New York, 1969).

${ }^{17}$ G. Eilenberger, Z. Phys. 182, 427 (1965).
${ }^{18}$ E. Helfand and N. R. Werthamer, Phys. Rev. 147, 288 (1966).

${ }^{19}$ S. J. Williamson and L. E. Valby, Phys. Rev. Lett. 24, 1061 (1970),

${ }^{20}$ S. J. Williamson, Phys. Rev. B 2, 3545 (1970).

${ }^{21}$ P. C. Hohenberg and N. R. Werthamer, Phys. Rev. 153, 493 (1967).

${ }^{22}$ K. Takanaka, Prog. Theor. Phys. 46, 357 (1971).

${ }^{23}$ A. A. Abrikosov, Zh. Eksp. Teor. Fiz. 321442 (1957) [Sov. Phys. JETP 5, 1174 (1957)].

${ }^{24}$ J. Schelten, H. Ullmaier, and W. Schmatz, Phys. Status Solidi B 48, 619 (1971).

${ }^{25}$ A. Yaouanc and P. Dalmas de Réotier, Muon Spin Rotation, Relaxation, and Resonance: Applications to Condensed Matter, International Series of Monographs on Physics 147 (Oxford University Press, Oxford, 2011).

${ }^{26}$ E. H. Brandt, Rep. Prog. Phys. 58, 1465 (1995).

${ }^{27}$ J. F. Annett, Superconductivity, Superfluids, and Condensates (Oxford University Press, Oxford, 2004).

${ }^{28}$ G. Eilenberger, Phys. Rev. 153, 584 (1967).

${ }^{29}$ P.Miranović, M. Ichioka, and K. Machida, Phys. Rev. B 70, 104510 (2004).

${ }^{30}$ P. Belova, M. Safonchik, K. B. Traito, and E. Lähderanta, Phys. Rev. B 83, 104518 (2011).

${ }^{31}$ M. Abramowitz and I. A. Stegun, editors, Handbook of Mathematical Functions (Dover Publications, New York, 1970). 